\title{
Article \\ Time-Inhomogeneous Feller-Type Diffusion Process in Population Dynamics
}

\author{
Virginia Giorno $^{+}(\mathbb{D})$ and Amelia G. Nobile $*,+(\mathbb{C}$ \\ Dipartimento di Informatica, Università degli Studi di Salerno, Via Giovanni Paolo II n. 132, \\ 84084 Fisciano, Salerno, Italy; giorno@unisa.it \\ * Correspondence: nobile@unisa.it \\ + These authors contributed equally to this work.
}

Citation: Giorno, V.; Nobile, A.G. Time-Inhomogeneous Feller-Type Diffusion Process in Population Dynamics. Mathematics 2021, 9, 1879. https://doi.org/10.3390/ math9161879

Academic Editor: Alexander Zeifman

Received: 29 June 2021

Accepted: 3 August 2021

Published: 7 August 2021

Publisher's Note: MDPI stays neutral with regard to jurisdictional claims in published maps and institutional affiliations.

Copyright: (C) 2021 by the authors. Licensee MDPI, Basel, Switzerland. This article is an open access article distributed under the terms and conditions of the Creative Commons Attribution (CC BY) license (https:// creativecommons.org/licenses/by/ $4.0 /)$.
Abstract: The time-inhomogeneous Feller-type diffusion process, having infinitesimal drift $\alpha(t) x+$ $\beta(t)$ and infinitesimal variance $2 r(t) x$, with a zero-flux condition in the zero-state, is considered. This process is obtained as a continuous approximation of a birth-death process with immigration. The transition probability density function and the related conditional moments, with their asymptotic behaviors, are determined. Special attention is paid to the cases in which the intensity functions $\alpha(t)$, $\beta(t), r(t)$ exhibit some kind of periodicity due to seasonal immigration, regular environmental cycles or random fluctuations. Various numerical computations are performed to illustrate the role played by the periodic functions.

Keywords: diffusion approximation; transient and asymptotic densities; conditional moments; periodic intensity functions

MSC: 60J60; 60J70; 92D25

\section{Introduction and Background}

One-dimensional diffusion processes are used to model the time evolution of dynamical systems in biology, genetics, physics, engineering, neuroscience, economics, finance, queueing and other fields (cf. for instance, Bharucha-Reid [1], Cox and Miller [2], Ricciardi [3,4], Tuckwell [5], Gardiner [6]). For many applications, it is often useful to consider the class of time-inhomogeneous linear diffusion processes, that includes the Feller-type diffusion process and the Ornstein-Uhlenbeck diffusion process. In this paper, we focus on the time-inhomogeneous Feller-type diffusion process with a zero-flux condition in the zero-state.

The Feller-type diffusion process $\left\{X(t), t \geq t_{0}\right\}, t_{0} \geq 0$, is an one-dimensional timeinhomogeneous diffusion process with linear infinitesimal drift and linear infinitesimal variance

$$
A_{1}(x, t)=\alpha(t) x+\beta(t), \quad A_{2}(x, t)=2 r(t) x,
$$

defined in the state-space $[0,+\infty)$, with $\alpha(t) \in \mathbb{R}, \beta(t) \geq 0, r(t)>0$ continuous functions for all $t \geq t_{0}$. Hence, $X(t)$ satisfies the following stochastic differential equation:

$$
d X(t)=[\alpha(t) X(t)+\beta(t)] d t+\sqrt{2 r(t) X(t)} d W(t), \quad X\left(t_{0}\right)=x_{0},
$$

where $W(t)$ is a standard Wiener process.

Feller diffusion process is widely used in population dynamics to model the growth of a population (cf. Feller [7], Ricciardi et al. [8], Pugliese and Milner [9], Masoliver and Perelló [10], Masoliver [11]). Indeed, in population dynamics the Feller-type diffusion process arises as a continuous approximation of a birth-death process with immigration. In these cases $\alpha(t)$, related to the growth intensity function, can be positive, negative or zero 
at different time instants. In particular, $\alpha(t)$ is positive (negative) when the birth intensity function is greater (less) than the death intensity function, whereas $\alpha(t)=0$ if the birth intensity function is equal to the death intensity function. Instead, the function $\beta(t)$ is related to the immigration intensity function. In particular, $\beta(t)>0$ indicates the presence of immigrations, whereas $\beta(t)=0$ denotes the absence of the immigration phenomena. The function $r(t)$ takes into account the environmental fluctuations and describes the noise intensity.

The Feller diffusion process is also used in queueing systems to describe the number of customers in a queue (cf. Di Crescenzo and Nobile [12]), in neurobiology to analyze the input-output behavior of single neurons (see, for instance, Sacerdote [13], Ditlevsen and Lánský [14], Buonocore et al. [15], Giorno et al. [16], Nobile and Pirozzi [17], D'Onofrio et al. [18]), in mathematical finance to model asset prices, market indices, interest rates and stochastic volatility (see, Tian and Zhang [19], Cox et al. [20], Maghsoodi [21], Peng and Schellhorn [22], Linetsky [23], Di Nardo and D'Onofrio [24]).

In many real applications, the transition probability density function (pdf) plays a relevant role for the description of the evolution of the dynamic system. In the sequel, we assume that a zero-flux condition is placed in the zero-state of $X(t)$ to ensure that the total probability mass is conserved in $[0,+\infty)$.

The transition pdf $f\left(x, t \mid x_{0}, t_{0}\right)$ of $X(t)$ is solution of Fokker-Planck equation

$$
\frac{\partial f\left(x, t \mid x_{0}, t_{0}\right)}{\partial t}=-\frac{\partial}{\partial x}\left\{[\alpha(t) x+\beta(t)] f\left(x, t \mid x_{0}, t_{0}\right)\right\}+r(t) \frac{\partial^{2}}{\partial x^{2}}\left[x f\left(x, t \mid x_{0}, t_{0}\right)\right],
$$

to solve with the initial delta condition

$$
\lim _{t \downarrow t_{0}} f\left(x, t \mid x_{0}, t_{0}\right)=\delta\left(x-x_{0}\right)
$$

and a zero-flux condition in the zero-state:

$$
\lim _{x \downarrow 0}\left\{[\alpha(t) x+\beta(t)] f\left(x, t \mid x_{0}, t_{0}\right)-r(t) \frac{\partial}{\partial x}\left[x f\left(x, t \mid x_{0}, t_{0}\right)\right]\right\}=0 .
$$

Indeed, denoting by

$$
\begin{aligned}
j\left(x, t \mid x_{0}, t_{0}\right) & =A_{1}(x, t) f\left(x, t \mid x_{0}, t_{0}\right)-\frac{1}{2} \frac{\partial}{\partial x}\left[A_{2}(x, t) f\left(x, t \mid x_{0}, t_{0}\right)\right] \\
& =[\alpha(t) x+\beta(t)] f\left(x, t \mid x_{0}, t_{0}\right)-r(t) \frac{\partial}{\partial x}\left[x f\left(x, t \mid x_{0}, t_{0}\right)\right], \quad x \geq 0
\end{aligned}
$$

the probability flux (or current) of $X(t)$, the Fokker-Planck Equation (2) can be re-written as $\partial f / \partial t=-\partial j / \partial x$ and the zero-flux condition (4) corresponds to requiring that $\int_{0}^{+\infty} f\left(x, t \mid x_{0}, t_{0}\right)$ $d x=1$ for all $t \geq t_{0}$.

We remark that the time-inhomogeneous Feller-type diffusion process with an absorbing boundary at the zero-state is considered in Lavigne and Roques [25] and in Giorno and Nobile [26], in which the first-passage time problem through the zero-state is also analyzed.

\section{Plan of the Paper}

The paper is organized in five sections and six appendices in which the proofs of the main results are reported. In Section 2, starting from the forward equations for the transition probabilities of the time-inhomogeneous birth-death process with immigration, we describe the continuous approximation that leads to the Fokker-Planck Equation (2), with the initial condition (3) and the zero-flux condition in the zero-state (4). In Section 3, for the time-inhomogeneous Feller-type diffusion process $X(t)$, we give some preliminary results concerning the moment generating function of the transition $\operatorname{pdf} f\left(x, t \mid x_{0}, t_{0}\right)$. Some special situations are analyzed: (i) the absence of immigration with $\beta(t)=0$, (ii) the proportional case in which $\beta(t)=\xi r(t)$, with $\xi>0$, and (iii) the time-homogeneous case. 
Sections 4 and 5 contain the main results of the paper concerning the analysis of transient and asymptotic behavior of the Feller-type diffusion process in the general case. Specifically, in Section 4 , the transition pdf $f\left(x, t \mid x_{0}, t_{0}\right)$ is obtained for the time-inhomogeneous Fellertype process in the general case for $x_{0}=0$ (Section 4.1) and for $x_{0}>0$ (Section 4.2). Finally, in Section 5, particular attention is paid to the periodic cases by assuming that the growth intensity function $\alpha(t)$, the immigration intensity function $\beta(t)$ and the noise intensity function $r(t)$ have some kind of periodicity. The asymptotic behaviors of the transition pdf and of the moments are also discussed in the following cases: periodic immigration intensity function, periodic growth intensity function, periodic immigration and growth intensity functions and periodic immigration, growth and noise intensity functions. Various numerical computations are performed making use of MATHEMATICA to analyze the role played by the involved periodic functions. Specifically, for some choices of the periodic functions $\alpha(t), \beta(t)$ and $r(t)$, of the interest in population dynamics, the transition densities, the conditional means and variances and their asymptotic behaviors are discussed and compared.

\section{Diffusion Approximation of Birth-Death Process with Immigration}

In this section, we show that the Feller-type diffusion process $X(t)$ can be obtained starting from a linear time-inhomogeneous birth-death process $N(t)$ with immigration by using a standard limit procedure (cf. for instance, Bhattacharya and Waymire [27]). Specifically, we prove that, under suitable assumptions, the discrete scaled process converges weakly to $X(t)$.

Let $\left\{N(t), t \geq t_{0}\right\}$ be a time-inhomogeneous linear birth-death process with immigration having state-space $\mathbb{N}_{0}$, conditioned to start from $j \in \mathbb{N}_{0}$ at time $t_{0}$. The transition probabilities of $N(t)$ satisfy the Kolmogorov forward equations and the related initial condition:

$$
\begin{aligned}
& \frac{d p_{j, 0}\left(t \mid t_{0}\right)}{d t}=-v(t) p_{j, 0}\left(t \mid t_{0}\right)+\mu(t) p_{j, 1}\left(t \mid t_{0}\right), \\
& \frac{d p_{j, n}\left(t \mid t_{0}\right)}{d t}=[\lambda(t)(n-1)+v(t)] p_{j, n-1}\left(t \mid t_{0}\right)+\mu(t)(n+1) p_{j, n+1}\left(t \mid t_{0}\right) \\
& -\{[\lambda(t)+\mu(t)] n+v(t)\} p_{j, n}\left(t \mid t_{0}\right), \quad n \in \mathbb{N}, \\
& \lim _{t \downarrow t_{0}} p_{j, n}\left(t \mid t_{0}\right)=\delta_{j, n}
\end{aligned}
$$

where $\lambda(t)>0, \mu(t)>0$ and $v(t) \geq 0$ are bounded and continuous functions for $t \geq t_{0}$ representing birth, death and immigration intensity functions, respectively, and $\delta_{j, n}$ is the Kronecker delta function. For $t \geq t_{0}$ and $j \in \mathbb{N}_{0}$, the probability generating function of the process $N(t)$ is (cf. Giorno and Nobile [28]):

$$
\begin{gathered}
G\left(z, t \mid j, t_{0}\right)=\sum_{n=0}^{+\infty} z^{n} p_{j, n}\left(t \mid t_{0}\right)=\left[\frac{1+(z-1) e^{\Lambda\left(t \mid t_{0}\right)-M\left(t \mid t_{0}\right)}\left[1-H\left(t \mid t_{0}\right)\right]}{1-(z-1) e^{\Lambda\left(t \mid t_{0}\right)-M\left(t \mid t_{0}\right)} H\left(t \mid t_{0}\right)}\right]^{j} \\
\quad \times \exp \left\{(z-1) \int_{t_{0}}^{t} \frac{v(u) e^{\Lambda(t \mid u)-M(t \mid u)}}{1-(z-1) e^{\Lambda\left(t \mid t_{0}\right)-M\left(t \mid t_{0}\right)}\left[H\left(t \mid t_{0}\right)-H\left(u \mid t_{0}\right)\right]} d u\right\},
\end{gathered}
$$

where

$$
\begin{aligned}
& \Lambda\left(t \mid t_{0}\right)=\int_{t_{0}}^{t} \lambda(\tau) d \tau, \quad M\left(t \mid t_{0}\right)=\int_{t_{0}}^{t} \mu(\tau) d \tau, \\
& H\left(t \mid t_{0}\right)=\int_{t_{0}}^{t} \lambda(\tau) e^{M\left(\tau \mid t_{0}\right)-\Lambda\left(\tau \mid t_{0}\right)} d \tau .
\end{aligned}
$$


By virtue of (7), the transition probabilities $p_{j, n}\left(t \mid t_{0}\right)$ are obtained in Giorno and Nobile [28]. Furthermore, for $t \geq t_{0}$ and $j \in \mathbb{N}_{0}$, the conditional mean and the conditional variance of $N(t)$ are

$$
\begin{aligned}
& \mathrm{E}\left[N(t) \mid N\left(t_{0}\right)=j\right]=j e^{\Lambda\left(t \mid t_{0}\right)-M\left(t \mid t_{0}\right)}+\int_{t_{0}}^{t} v(u) e^{\Lambda(t \mid u)-M(t \mid u)} d u, \\
& \operatorname{Var}\left[N(t) \mid N\left(t_{0}\right)=j\right]=j e^{\Lambda\left(t \mid t_{0}\right)-M\left(t \mid t_{0}\right)}\left\{1-e^{\Lambda\left(t \mid t_{0}\right)-M\left(t \mid t_{0}\right)}\right. \\
& \left.\quad+2 e^{\Lambda\left(t \mid t_{0}\right)-M\left(t \mid t_{0}\right)} H\left(t \mid t_{0}\right)\right\}+\int_{t_{0}}^{t} v(u) e^{\Lambda(t \mid u)-M(t \mid u)} d u \\
& \quad+2 e^{\Lambda\left(t \mid t_{0}\right)-M\left(t \mid t_{0}\right)} \int_{t_{0}}^{t} v(u) e^{\Lambda(t \mid u)-M(t \mid u)}\left[H\left(t \mid t_{0}\right)-H\left(u \mid t_{0}\right)\right] d u,
\end{aligned}
$$

with $\Lambda\left(t \mid t_{0}\right), M\left(t \mid t_{0}\right)$ and $H\left(t \mid t_{0}\right)$ defined in (8).

We now consider a diffusion approximation of $N(t)$. To this purpose, we use a suitable procedure, similar to that adopted in Di Crescenzo et al. [29,30] and Dharmaraja et al. [31]. We rename the intensity functions related to $N(t)$, by setting

$$
\lambda(t)=\alpha_{1}(t)+\frac{r(t)}{\epsilon}, \quad \mu(t)=\alpha_{2}(t)+\frac{r(t)}{\epsilon}, \quad v(t)=\frac{\beta(t)}{\epsilon},
$$

where $\epsilon$ is a positive scaling parameter. In (10), $\alpha_{1}(t)>0, \alpha_{2}(t)>0, r(t)>0$ and $\beta(t) \geq 0$ are bounded and continuous functions for $t \geq t_{0}$. Let us consider the Markov process $\left\{N_{\epsilon}(t), t \geq t_{0}\right\}$, with $N_{\epsilon}(t)=\epsilon N(t)$, having state-space $\{0, \epsilon, 2 \epsilon, \ldots\}$. For $\varepsilon \downarrow 0$, the scaled process $N_{\epsilon}(t)$ converges weakly to a diffusion process $\left\{X(t), t \geq t_{0}\right\}$ having state-space $[0,+\infty)$. Indeed, with reference to the system (6), substituting $p_{j, n}\left(t \mid t_{0}\right)$ with $f\left(n \varepsilon, t \mid j \varepsilon, t_{0}\right) \varepsilon$ and setting $x=n \epsilon$ and $x_{0}=j \varepsilon$, we have:

$$
\begin{gathered}
\frac{\partial f\left(x, t \mid x_{0}, t_{0}\right)}{\partial t}=\frac{\lambda(t)(x-\varepsilon)+v(t) \varepsilon}{\epsilon} f\left(x-\varepsilon, t \mid x_{0}, t_{0}\right) \\
-\frac{[\lambda(t)+\mu(t)] x+v(t) \varepsilon}{\epsilon} f\left(x, t \mid x_{0}, t_{0}\right)+\frac{\mu(t)(x+\varepsilon)}{\epsilon} f\left(x+\varepsilon, t \mid x_{0}, t_{0}\right), \\
\lim _{x \rightarrow 0}\left\{\epsilon \frac{\partial f\left(x, t \mid x_{0}, t_{0}\right)}{\partial t}+[\lambda(t) x+v(t) \varepsilon] f\left(x, t \mid x_{0}, t_{0}\right)\right. \\
\left.-\mu(t)(x+\varepsilon) f\left(x+\varepsilon, t \mid x_{0}, t_{0}\right)\right\}=0,
\end{gathered}
$$

with the intensity functions $\lambda(t), \mu(t)$ and $v(t)$ defined in (10). Expanding $f\left(x-\varepsilon, t \mid x_{0}, t_{0}\right)$ and $f\left(x+\varepsilon, t \mid x_{0}, t_{0}\right)$ as Taylor series, taking the limit as $\varepsilon \downarrow 0$ and setting $\alpha(t)=\alpha_{1}(t)-$ $\alpha_{2}(t)$, we obtain (2) and (4) with the delta initial condition (3). Hence, making use of the considered approximating procedure, the Fokker-Planck Equation (2) can be obtained from the second of (6), whereas the zero-flux condition (4) can be derived from the first equation of (6). Moreover, from (10), it follows that $\alpha(t)=\lambda(t)-\mu(t)$, so that $\alpha(t)$ can be positive, negative or null for each fixed $t$.

\section{Moment Generating Function and Transition PDF in Special Cases}

Let $\left\{X(t), t \geq t_{0}\right\}, t_{0} \geq 0$, be the time-inhomogeneous Feller-type diffusion process with infinitesimal drift and infinitesimal variance given in (1), defined in the state-space $[0,+\infty)$, with a zero-flux condition in the zero-state. In this section, we determine the moment generating function, the conditional mean and the conditional variance. Furthermore, the explicit expression of the transition pdf $f\left(x, t \mid x_{0}, t_{0}\right)$ is obtained in the following special situations: (i) in the absence of immigration, (ii) for $\beta(t)=\xi r(t)$, with $\xi>0$, and (iii) for the time-homogeneous process. 


\subsection{Moment Generating Function, Conditional Mean and Conditional Variance}

For $t \geq t_{0}$ and $x_{0} \geq 0$, we consider the moment generating function:

$$
M\left(s, t \mid x_{0}, t_{0}\right)=\int_{0}^{+\infty} e^{-s x} f\left(x, t \mid x_{0}, t_{0}\right) d x, \quad \operatorname{Re} s>0
$$

Multiplying both sides of (2) by $e^{-s x}$, integrating with respect to $x$ over the interval $[0,+\infty)$ and making use of the boundary condition (4), we obtain the following partial differential equation

$$
\frac{\partial M\left(s, t \mid x_{0}, t_{0}\right)}{\partial t}-s[\alpha(t)-s r(t)] \frac{\partial M\left(s, t \mid x_{0}, t_{0}\right)}{\partial s}+s \beta(t) M\left(s, t \mid x_{0}, t_{0}\right)=0,
$$

to solve with the initial condition

$$
\lim _{t \downarrow t_{0}} M\left(s, t \mid x_{0}, t_{0}\right)=e^{-s x_{0}}
$$

derived from (11) by using the initial condition (3).

Proposition 1. For $t \geq t_{0}$, by assuming that $\alpha(t) \in \mathbb{R}, \beta(t) \geq 0$ and $r(t)>0$, the moment generating function of the Feller-type diffusion process $X(t)$ with a zero-flux condition in the zero-state is:

$$
\begin{aligned}
M\left(s, t \mid x_{0}, t_{0}\right)=\exp & \left\{-s \int_{t_{0}}^{t} \frac{\beta(u) e^{A(t \mid u)}}{1+s e^{A\left(t \mid t_{0}\right)}\left[R\left(t \mid t_{0}\right)-R\left(u \mid t_{0}\right)\right]} d u\right\} \\
& \times \exp \left\{-\frac{s x_{0} e^{A\left(t \mid t_{0}\right)}}{1+s e^{A\left(t \mid t_{0}\right)} R\left(t \mid t_{0}\right)}\right\}, \quad x_{0} \geq 0,
\end{aligned}
$$

where

$$
A\left(t \mid t_{0}\right)=\int_{t_{0}}^{t} \alpha(z) d z, \quad R\left(t \mid t_{0}\right)=\int_{t_{0}}^{t} r(\tau) e^{-A\left(\tau \mid t_{0}\right)} d \tau
$$

Proof. The proof is given in Appendix A.

The expression of the moment generating function, given in (14), allows to determine the conditional mean and the conditional variance of the time-inhomogeneous Feller-type diffusion process $X(t)$. Indeed, for $t \geq t_{0}$ and $x_{0} \geq 0$ one has:

$$
\begin{aligned}
\mathrm{E}\left[X(t) \mid X\left(t_{0}\right)=x_{0}\right]= & x_{0} e^{A\left(t \mid t_{0}\right)}+\int_{t_{0}}^{t} \beta(u) e^{A(t \mid u)} d u, \\
\operatorname{Var}\left[X(t) \mid X\left(t_{0}\right)=x_{0}\right]= & 2 x_{0} e^{2 A\left(t \mid t_{0}\right)} R\left(t \mid t_{0}\right) \\
& +2 e^{A\left(t \mid t_{0}\right)} \int_{t_{0}}^{t} \beta(u) e^{A(t \mid u)}\left[R\left(t \mid t_{0}\right)-R\left(u \mid t_{0}\right)\right] d u .
\end{aligned}
$$

We note that the conditional mean in (16) coincides with the solution of the linear first-order differential equation:

$$
\frac{d x(t)}{d t}=\alpha(t) x(t)+\beta(t), \quad x\left(t_{0}\right)=x_{0}
$$


Moreover, Equations (14) and (16) can be also derived from (7) and (9), respectively, making use of the diffusion approximation described in Section 2. Indeed, by virtue of (10) with $\alpha(t)=\alpha_{1}(t)-\alpha_{2}(t)$, from (7) and (9) one has:

$$
\begin{aligned}
& M\left(s, t \mid x_{0}, t_{0}\right)=\lim _{\varepsilon \downarrow 0} G\left(e^{-s \varepsilon}, t \mid \frac{x_{0}}{\varepsilon}, t_{0}\right), \\
& \mathrm{E}\left[X(t) \mid X\left(t_{0}\right)=x_{0}\right]=\lim _{\varepsilon \downarrow 0}\left\{\varepsilon \mathrm{E}\left[N(t) \mid N\left(t_{0}\right)=\frac{x_{0}}{\varepsilon}\right]\right\}, \\
& \operatorname{Var}\left[X(t) \mid X\left(t_{0}\right)=x_{0}\right]=\lim _{\varepsilon \downarrow 0}\left\{\varepsilon^{2} \operatorname{Var}\left[N(t) \mid N\left(t_{0}\right)=\frac{x_{0}}{\varepsilon}\right]\right\} .
\end{aligned}
$$

\subsection{Absence of Immigration}

We assume that the immigration intensity function $\beta(t)=0, \alpha(t) \in \mathbb{R}$ and $r(t)>0$ for $t \geq 0$.

Proposition 2. If $\beta(t)=0$, for $t \geq t_{0}$ the moment generating function (14) becomes:

$$
M^{*}\left(s, t \mid x_{0}, t_{0}\right)=\exp \left\{-\frac{s x_{0} e^{A\left(t \mid t_{0}\right)}}{1+s e^{A\left(t \mid t_{0}\right)} R\left(t \mid t_{0}\right)}\right\}, \quad x_{0} \geq 0 .
$$

Furthermore, the transition $p d f$ of $X(t)$ with a zero-flux condition in the zero-state is:

$$
f^{*}\left(x, t \mid x_{0}, t_{0}\right)= \begin{cases}\delta(x), & x_{0}=0, \\ \exp \left\{-\frac{x_{0}}{R\left(t \mid t_{0}\right)}\right\} \delta(x)+\frac{1}{R\left(t \mid t_{0}\right)} \sqrt{\frac{x_{0} e^{-A\left(t \mid t_{0}\right)}}{x}} & \\ \quad \quad \quad \exp \left\{-\frac{x_{0}+x e^{-A\left(t \mid t_{0}\right)}}{R\left(t \mid t_{0}\right)}\right\} I_{1}\left[\frac{2 \sqrt{x x_{0} e^{-A\left(t \mid t_{0}\right)}}}{R\left(t \mid t_{0}\right)}\right], & x_{0}>0\end{cases}
$$

with $A\left(t \mid t_{0}\right)$ and $R\left(t \mid t_{0}\right)$ defined in (15) and where

$$
I_{v}(z)=\sum_{k=0}^{+\infty} \frac{1}{k ! \Gamma(v+k+1)}\left(\frac{z}{2}\right)^{2 k+v}, \quad v \in \mathbb{R}
$$

denotes the modified Bessel function of the first kind and $\Gamma(\xi)$ is the Eulero gamma function.

Proof. The proof is given in Appendix B.

Equation (18) is in agreement with the expression given in Masoliver [11] and in Gan and Waxman [32]. We now consider the random variable $T\left(x_{0}, t_{0}\right)$ describing the first-passage time through the zero-state starting from $x_{0}>0$ at time $t_{0}$. We note that (18) can be rewritten as

$$
f^{*}\left(x, t \mid x_{0}, t_{0}\right)=P\left\{T\left(x_{0}, t_{0}\right)<t\right\} \delta(x)+f_{a}\left(x, t \mid x_{0}, t_{0}\right), \quad x_{0}>0,
$$

where

$$
P\left\{T\left(x_{0}, t_{0}\right)<t\right\}=\exp \left\{-\frac{x_{0}}{R\left(t \mid t_{0}\right)}\right\}
$$

is the first-passage time probability through the zero-state starting from $x_{0}>0$ and $f_{a}\left(x, t \mid x_{0}, t_{0}\right)$ denotes the transition pdf of the considered Feller process in the presence of an absorbing boundary in the zero-state (cf. for instance, Giorno and Nobile [26]):

$$
f_{a}\left(x, t \mid x_{0}, t_{0}\right)=\frac{1}{R\left(t \mid t_{0}\right)} \sqrt{\frac{x_{0} e^{-A\left(t \mid t_{0}\right)}}{x}} \exp \left\{-\frac{x_{0}+x e^{-A\left(t \mid t_{0}\right)}}{R\left(t \mid t_{0}\right)}\right\} I_{1}\left[\frac{2 \sqrt{x x_{0} e^{-A\left(t \mid t_{0}\right)}}}{R\left(t \mid t_{0}\right)}\right] .
$$


From (18) it follows:

$$
\int_{0}^{+\infty} f^{*}\left(x, t \mid x_{0}, t_{0}\right) d x=P\left\{T\left(x_{0}, t_{0}\right)<t\right\}+\int_{0}^{+\infty} f_{a}\left(x, t \mid x_{0}, t_{0}\right) d x=1 .
$$

Setting $\beta(t)=0$ in (16), we obtain the conditional mean and the conditional variance of $X(t)$ in the absence of immigration. Moreover, making use of (18), the $k$-th conditional moment can be evaluated:

$$
\begin{aligned}
\mathrm{E}\left[X^{k}(t) \mid X\left(t_{0}\right)=x_{0}\right]=\int_{0}^{+\infty} x^{k} f_{X}^{*}\left(x, t \mid x_{0}, t_{0}\right) d x \\
=(k-1) !\left[R\left(t \mid t_{0}\right) e^{A\left(t \mid t_{0}\right)}\right]^{k} \sum_{j=1}^{k}\left(\begin{array}{l}
k \\
j
\end{array}\right) \frac{1}{(j-1) !}\left[\frac{x_{0}}{R\left(t \mid t_{0}\right)}\right]^{j}, \quad k=1,2, \ldots
\end{aligned}
$$

We note that if $x_{0}=0$, from (20) one has $\mathrm{E}\left[X^{k}(t) \mid X\left(t_{0}\right)=0\right]=0$, according to the first expression of (18).

\subsection{Proportional Case}

For $t \geq 0$, we assume that the functions $\beta(t)$ and $r(t)$ are proportional:

$$
\beta(t)=\xi r(t), \quad \xi>0 .
$$

Proposition 3. Under the assumption (21), if $\alpha(t) \in \mathbb{R}$ and $\beta(t)>0$ for $t \geq t_{0}$ one has:

$$
M\left(s, t \mid x_{0}, t_{0}\right)=\left[1+s e^{A\left(t \mid t_{0}\right)} R\left(t \mid t_{0}\right)\right]^{-\xi} \exp \left\{-\frac{s x_{0} e^{A\left(t \mid t_{0}\right)}}{1+s e^{A\left(t \mid t_{0}\right)} R\left(t \mid t_{0}\right)}\right\}, \quad x_{0} \geq 0 .
$$

Furthermore, the transition $p d f$ of $X(t)$ with a zero-flux condition in the zero-state is:

$$
f\left(x, t \mid x_{0}, t_{0}\right)= \begin{cases}\frac{1}{x \Gamma(\xi)}\left[\frac{x e^{-A\left(t \mid t_{0}\right)}}{R\left(t \mid t_{0}\right)}\right]^{\xi} \exp \left\{-\frac{x e^{-A\left(t \mid t_{0}\right)}}{R\left(t \mid t_{0}\right)}\right\}, & x_{0}=0, \\ \frac{e^{-A\left(t \mid t_{0}\right)}}{R\left(t \mid t_{0}\right)}\left[\frac{x e^{-A\left(t \mid t_{0}\right)}}{x_{0}}\right]^{(\xi-1) / 2} \exp \left\{-\frac{x_{0}+x e^{-A\left(t \mid t_{0}\right)}}{R\left(t \mid t_{0}\right)}\right\} & \\ \quad \times I_{\xi-1}\left[\frac{2 \sqrt{x x_{0} e^{-A\left(t \mid t_{0}\right)}}}{R\left(t \mid t_{0}\right)}\right], & x_{0}>0,\end{cases}
$$

with $A\left(t \mid t_{0}\right)$ and $R\left(t \mid t_{0}\right)$ defined in (15) and $I_{v}(z)$ given in (19).

Proof. The proof is given in Appendix C.

Since for fixed $v$, when $z \rightarrow 0$ (cf. Abramowitz and Stegun [33], p. 375, no 9.6.7)

$$
I_{v}(z) \sim \frac{1}{\Gamma(v+1)}\left(\frac{z}{2}\right)^{v} \quad v \neq-1,-2, \ldots,
$$

the first formula of (23) follows from the second expression as $x_{0} \downarrow 0$. Furthermore, by virtue of (24), from (23) for $x_{0} \geq 0$ one has:

$$
\lim _{x \downarrow 0} f\left(x, t \mid x_{0}, t_{0}\right)= \begin{cases}+\infty, & 0<\xi<1, \\ \frac{e^{-A\left(t \mid t_{0}\right)}}{R\left(t \mid t_{0}\right)} \exp \left\{-\frac{x_{0}}{R\left(t \mid t_{0}\right)}\right\}, & \xi=1, \\ 0, & \xi>1 .\end{cases}
$$

Relation (25) shows that if $\xi>1$ the zero-state behaves as an entrance boundary that cannot be reached from the interior of the state-space, while it is possible to starts right there. 
By setting $\beta(t)=\xi r(t)$, with $\xi>0$, in (16), we obtain the conditional mean and the conditional variance of $X(t)$ in the proportional case. Moreover, making use of (23), the $k$-th conditional moment can be evaluated:

$$
\begin{aligned}
\mathrm{E}\left[X^{k}(t) \mid X\left(t_{0}\right)\right. & \left.=x_{0}\right]=k !\left[R\left(t \mid t_{0}\right) e^{A\left(t \mid t_{0}\right)}\right]^{k}\left\{\frac{(\xi)_{k}}{k !}\right. \\
+ & \left.\sum_{i=1}^{k} \frac{(\xi)_{k-i}}{i(k-i) !} \sum_{j=1}^{i}\left(\begin{array}{l}
i \\
j
\end{array}\right) \frac{1}{(j-1) !}\left[\frac{x_{0}}{R\left(t \mid t_{0}\right)}\right]^{j}\right\}, \quad k=1,2, \ldots,
\end{aligned}
$$

where $(\xi)_{n}$ denotes the Pochhammer symbol, defined as $(\xi)_{0}=1$ and $(\xi)_{n}=\xi(\xi+$ $1) \cdots(\xi+n-1)$ for $n=1,2, \ldots$ In particular, by setting $x_{0}=0$ in (26) one has:

$$
\mathrm{E}\left[X^{k}(t) \mid X\left(t_{0}\right)=0\right]=(\xi)_{k}\left[R\left(t \mid t_{0}\right) e^{A\left(t \mid t_{0}\right)}\right]^{k}, \quad k=1,2 \ldots
$$

\subsection{Time-Homogeneous Feller Process}

We consider the time-homogeneous Feller process, obtained from (1) by setting $\alpha(t)=$ $\alpha, \beta(t)=\beta, r(t)=r$, with $\alpha \in \mathbb{R}, \beta \geq 0$ and $r>0$. This process is analyzed by Feller [34,35]. The explicit expression of transition pdf in the presence of a zero-flux condition in the zero-state for $\beta>0$ is given in Karlin and Taylor [36] and in Giorno et al. [37]. From (15) we have:

$$
A\left(t \mid t_{0}\right)=\alpha\left(t-t_{0}\right), \quad R\left(t \mid t_{0}\right)= \begin{cases}r\left(t-t_{0}\right), & \alpha=0, \\ \frac{r}{\alpha}\left(1-e^{-\alpha\left(t-t_{0}\right)}\right), & \alpha \neq 0 .\end{cases}
$$

In the absence of immigration, i.e., when $\beta=0$, the transition pdf can be obtained from (18) making use of (27). When $\alpha=0, \beta>0$ and $r>0$, by virtue of (27), one has:

$$
f\left(x, t \mid x_{0}, t_{0}\right)=\left\{\begin{array}{cc}
\frac{1}{x \Gamma(\beta / r)}\left[\frac{x}{r\left(t-t_{0}\right)}\right]^{\beta / r} \exp \left\{-\frac{x}{r\left(t-t_{0}\right)}\right\}, & x_{0}=0, \\
\frac{1}{r\left(t-t_{0}\right)}\left(\frac{x}{x_{0}}\right)^{(\beta-r) /(2 r)} \exp \left\{-\frac{x_{0}+x}{r\left(t-t_{0}\right)}\right\} & \\
\times I_{\beta / r-1}\left[\frac{2 \sqrt{x x_{0}}}{r\left(t-t_{0}\right)}\right], & x_{0}>0,
\end{array}\right.
$$

whereas if $\alpha \neq 0, \beta>0$ and $r>0$ one obtains:

$$
f\left(x, t \mid x_{0}, t_{0}\right)= \begin{cases}\frac{1}{x \Gamma(\beta / r)}\left[\frac{\alpha x}{r\left(e^{\alpha\left(t-t_{0}\right)}-1\right)}\right]^{\beta / r} \exp \left\{-\frac{\alpha x}{r\left(e^{\alpha\left(t-t_{0}\right)}-1\right)}\right\}, & x_{0}=0, \\ \frac{\alpha}{r\left(e^{\alpha\left(t-t_{0}\right)}-1\right)}\left[\frac{x e^{-\alpha\left(t-t_{0}\right)}}{x_{0}}\right]^{(\beta-r) /(2 r)} \exp \left\{-\frac{\alpha\left[x+x_{0} e^{\alpha\left(t-t_{0}\right)}\right]}{r\left(e^{\alpha\left(t-t_{0}\right)}-1\right)}\right\} & \\ \times I_{\beta / r-1}\left[\frac{2 \alpha \sqrt{x x_{0} e^{\alpha\left(t-t_{0}\right)}}}{r\left(e^{\left.\alpha^{\left(t-t_{0}\right.}\right)}-1\right)}\right], & x_{0}>0 .\end{cases}
$$

Note that (28) and (29) can be derived from (23) by setting $\xi=\beta / r, A\left(t \mid t_{0}\right)$ and $R\left(t \mid t_{0}\right)$ as in (27). Moreover, by carrying out the same choices in (26), the conditioned moments are also obtained.

When $\alpha<0, \beta>0$ and $r>0$, the time-homogeneous Feller process admits a steady-state behavior:

$$
W(x)=\lim _{t \rightarrow+\infty} f\left(x, t \mid x_{0}, t_{0}\right)=\frac{1}{x \Gamma(\beta / r)}\left(\frac{|\alpha| x}{r}\right)^{\beta / r} \exp \left\{-\frac{|\alpha| x}{r}\right\}, \quad x>0,
$$


that is a gamma density of parameters $\beta / r$ and $r /|\alpha|$. We note that

$$
\lim _{x \downarrow 0} W(x)= \begin{cases}+\infty, & \beta<r \\ |\alpha| / r, & \beta=r \\ 0, & \beta>r .\end{cases}
$$

The steady-state density $W(x)$ is a decreasing function of $x$ when $\beta \leq r$, whereas $W(x)$ has a single maximum in $x=(\beta-r) /|\alpha|$ for $\beta>r$. Furthermore, the asymptotic moments are:

$$
E\left(X^{k}\right)=\lim _{t \rightarrow+\infty} E\left[X^{k}(t) \mid X\left(t_{0}\right)=x_{0}\right]=\left(\frac{r}{|\alpha|}\right)^{k} \frac{\Gamma(k+\beta / r)}{\Gamma(\beta / r)}, \quad k=1,2, \ldots
$$

\section{Transition PDF and Conditional Moments in the General Case}

In this section, we obtain the transition pdf and its moments for the Feller-type diffusion process (1) with a zero-flux condition in the zero-state in the general case. Furthermore, the special cases considered in the Section 3 are now derived from the general case.

From (14), for $t \geq t_{0}$ we note that

$$
M\left(s, t \mid x_{0}, t_{0}\right)= \begin{cases}M\left(s, t \mid 0, t_{0}\right), & x_{0}=0, \\ M\left(s, t \mid 0, t_{0}\right) M^{*}\left(s, t \mid x_{0}, t_{0}\right), & x_{0}>0,\end{cases}
$$

where $M\left(s, t \mid 0, t_{0}\right)$ is the moment generating function of $X(t)$ for $x_{0}=0$, whereas $M^{*}\left(s, t \mid x_{0}, t_{0}\right)$ is the moment generating function given in (17).

Therefore, to determine the transition $\operatorname{pdf} f\left(x, t \mid x_{0}, t_{0}\right)$ of the time-inhomogeneous Feller process, we proceed as follows:

(1) we determine the transition pdf $f\left(x, t \mid 0, t_{0}\right)$ for $x \geq 0$ and $t \geq t_{0}$;

(2) we calculate the transition density $f\left(x, t \mid x_{0}, t_{0}\right)$ for $x_{0}>0, x \geq 0$ and $t \geq t_{0}$ as a convolution between $f\left(x, t \mid 0, t_{0}\right)$ and the transition pdf $f^{*}\left(x, t \mid x_{0}, t_{0}\right)$, given in (18), of the Feller-type process in the absence of immigration.

\subsection{General Case: $x_{0}=0$}

To determine the transition pdf of time-inhomogeneous Feller-type process with a zero-flux condition in the zero-state, we set $x_{0}=0$ in (14), so that for $t \geq t_{0}$ we obtain:

$$
M\left(s, t \mid 0, t_{0}\right)=\exp \left\{-s \int_{t_{0}}^{t} \frac{\beta(u) e^{A(t \mid u)}}{1+s e^{A\left(t \mid t_{0}\right)}\left[R\left(t \mid t_{0}\right)-R\left(u \mid t_{0}\right)\right]} d u\right\},
$$

with $A\left(t \mid t_{0}\right)$ and $R\left(t \mid t_{0}\right)$ defined in (15).

In the sequel, we denote by $B_{n}\left(d_{1}, d_{2}, \ldots, d_{n}\right)$ the complete Bell polynomials, recursively defined as follows:

$$
B_{0}=1, \quad B_{n+1}\left(d_{1}, d_{2}, \ldots, d_{n+1}\right)=\sum_{i=0}^{n}\left(\begin{array}{c}
n \\
i
\end{array}\right) B_{n-i}\left(d_{1}, d_{2}, \ldots, d_{n-i}\right) d_{i+1}, \quad n \in \mathbb{N}_{0}
$$

with

$$
d_{k}=-\frac{k !}{\left[R\left(t \mid t_{0}\right)\right]^{k}} \int_{t_{0}}^{t} \beta(u) e^{-A\left(u \mid t_{0}\right)}\left[R\left(u \mid t_{0}\right)\right]^{k-1} d u, \quad k=1,2, \ldots
$$

In particular, from (31) and (32) one has

$$
\begin{aligned}
B_{1}\left(d_{1}\right)=d_{1}=-\frac{1}{R\left(t \mid t_{0}\right)} \int_{t_{0}}^{t} \beta(u) e^{-A\left(u \mid t_{0}\right)} d u, & \\
B_{2}\left(d_{1}, d_{2}\right)=d_{1}^{2}+d_{2}= & \frac{1}{R^{2}\left(t \mid t_{0}\right)}\left\{\left[\int_{t_{0}}^{t} \beta(u) e^{-A\left(u \mid t_{0}\right)} d u\right]^{2}\right. \\
& \left.-2 \int_{t_{0}}^{t} \beta(u) e^{-A\left(u \mid t_{0}\right)} R\left(u \mid t_{0}\right) d u\right\} .
\end{aligned}
$$


Furthermore, we consider the Laguerre polynomials

$$
L_{n}(y)=\frac{e^{y}}{n !} \frac{d^{n}}{d y^{n}}\left(e^{-y} y^{n}\right)=\sum_{k=0}^{n}\left(\begin{array}{l}
n \\
k
\end{array}\right) \frac{(-1)^{k} y^{k}}{k !}, \quad n=0,1, \ldots
$$

whose derivative (cf. Gradshteyn and Ryzhik [38], p. 1001, n. 8.971.3) is:

$$
\frac{d L_{n}(y)}{d y}=\frac{n L_{n}(y)-n L_{n-1}(y)}{y}
$$

Proposition 4. Under the assumptions of Proposition 1 , for $t \geq t_{0}$ and $x_{0}=0$ the transition pdf of the time-inhomogeneous Feller-type diffusion process $X(t)$ with a condition of zero-flux in the zero-state is

$$
f\left(x, t \mid 0, t_{0}\right)=\sum_{n=0}^{+\infty} \frac{B_{n}\left(d_{1}, d_{2}, \ldots, d_{n}\right)}{n !} \delta(x)+\exp \left\{-\frac{x e^{-A\left(t \mid t_{0}\right)}}{R\left(t \mid t_{0}\right)}\right\} \Phi\left(x, t \mid t_{0}\right),
$$

where

$$
\Phi\left(z, t \mid t_{0}\right)=\sum_{n=1}^{+\infty} \frac{B_{n}\left(d_{1}, d_{2}, \ldots, d_{n}\right)}{n !} \frac{d}{d z} L_{n}\left[\frac{z e^{-A\left(t \mid t_{0}\right)}}{R\left(t \mid t_{0}\right)}\right], \quad z>0,
$$

with $A\left(t \mid t_{0}\right)$ and $R\left(t \mid t_{0}\right)$ defined in (15), $B_{n}\left(d_{1}, d_{2}, \ldots, d_{n}\right)$ given in (31) and (32), $L_{n}(y)$ and $d L_{n}(y) / d y$ defined in (34) and (35), respectively.

Proof. The proof is given in Appendix D.

Proposition 5. Under the assumptions of Proposition 1 , for $t \geq t_{0}$ the $k$-th conditional moment of $X(t)$ with $X\left(t_{0}\right)=0$ is:

$$
\mathrm{E}\left[X^{k}(t) \mid X\left(t_{0}\right)=0\right]=k !\left[R\left(t \mid t_{0}\right) e^{A\left(t \mid t_{0}\right)}\right]^{k} \sum_{n=1}^{k}(-1)^{n}\left(\begin{array}{l}
k-1 \\
n-1
\end{array}\right) \frac{B_{n}\left(d_{1}, d_{2}, \ldots, d_{n}\right)}{n !}
$$

for $k=1,2, \ldots$

Proof. The proof is given in Appendix E.

We note that, by virtue of (33), from (38) follows (16) for $x_{0}=0$.

\subsection{General Case: $x_{0}>0$}

We determine the transition pdf of $X(t)$ when $x_{0}>0$.

Proposition 6. For $t \geq t_{0}$ and $x_{0}>0$, the transition $p d f$ of the time-inhomogeneous Feller-type diffusion process $X(t)$ with a condition of zero-flux in the zero-state is:

$$
\begin{aligned}
f\left(x, t \mid x_{0}, t_{0}\right) & =f^{*}\left(x, t \mid x_{0}, t_{0}\right) \sum_{n=0}^{+\infty} \frac{B_{n}\left(d_{1}, d_{2}, \ldots, d_{n}\right)}{n !} \\
& +\exp \left\{-\frac{x_{0}+x e^{-A\left(t \mid t_{0}\right)}}{R\left(t \mid t_{0}\right)}\right\}\left\{\Phi\left(x, t \mid t_{0}\right)+\frac{1}{R\left(t \mid t_{0}\right)}\right. \\
& \left.\times \int_{0}^{x} \sqrt{\frac{x_{0} e^{-A\left(t \mid t_{0}\right)}}{x-z}} I_{1}\left[\frac{2 \sqrt{(x-z) x_{0} e^{-A\left(t \mid t_{0}\right)}}}{R\left(t \mid t_{0}\right)}\right] \Phi\left(z, t \mid t_{0}\right) d z\right\},
\end{aligned}
$$

where $f^{*}\left(x, t \mid x_{0}, t_{0}\right)$ is the transition pdf in the absence of immigration, defined in (18), and $\Phi\left(x, t \mid t_{0}\right)$ is given (37). 
Proof. For $x_{0}>0$, the moment generating function (14) for the Feller process $X(t)$ can be written as $M\left(s, t \mid x_{0}, t_{0}\right)=M\left(s, t \mid 0, t_{0}\right) M^{*}\left(s, t \mid x_{0}, t_{0}\right)$, where $M\left(s, t \mid 0, t_{0}\right)$ is the moment generating function of $X(t)$ for $x_{0}=0$, whereas $M^{*}\left(s, t \mid x_{0}, t_{0}\right)$ is the moment generating function given (17). Therefore, for $t \geq t_{0}$ the transition $\operatorname{pdf} f\left(x, t \mid x_{0}, t_{0}\right)$ is given by the following convolution:

$$
f\left(x, t \mid x_{0}, t_{0}\right)=\int_{0}^{x} f\left(x-z, t \mid 0, t_{0}\right) f^{*}\left(z, t \mid x_{0}, t_{0}\right) d z, \quad x_{0}>0
$$

from which, recalling (18) and (36), Equation (39) follows.

Proposition 7. Under the assumptions of Proposition 6, for $t \geq t_{0}$ the $k$-th conditional moment of $X(t)$ with $X\left(t_{0}\right)=x_{0}>0$ is:

$$
\begin{aligned}
& \mathrm{E}\left[X^{k}(t) \mid X\left(t_{0}\right)=x_{0}\right]=k !\left[R\left(t \mid t_{0}\right) e^{A\left(t \mid t_{0}\right)}\right]^{k}\left\{\sum_{n=1}^{k}(-1)^{n}\left(\begin{array}{l}
k-1 \\
n-1
\end{array}\right) \frac{B_{n}\left(d_{1}, d_{2}, \ldots, d_{n}\right)}{n !}\right. \\
& +\sum_{i=1}^{k-1} \frac{1}{i}\left[\sum_{j=1}^{i}\left(\begin{array}{l}
i \\
j
\end{array}\right) \frac{1}{(j-1) !}\left[\frac{x_{0}}{R\left(t \mid t_{0}\right)}\right]^{j}\right]\left[\sum_{n=1}^{k-i}(-1)^{n}\left(\begin{array}{c}
k-i-1 \\
n-1
\end{array}\right) \frac{B_{n}\left(d_{1}, d_{2}, \ldots, d_{n}\right)}{n !}\right] \\
& \left.+\frac{1}{k} \sum_{j=1}^{k}\left(\begin{array}{c}
k \\
j
\end{array}\right) \frac{1}{(j-1) !}\left[\frac{x_{0}}{R\left(t \mid t_{0}\right)}\right]^{j}\right\}
\end{aligned}
$$

for $k=2,3, \ldots$, whereas for $k=1$ the first formula of (16) holds.

Proof. The proof is given in Appendix F.

Note that (36) and (38) follow taking the limit as $x_{0} \downarrow 0$ in (39) and (41), respectively.

Remark 1 (Absence of immigration). We assume that $\beta(t)=0$. We prove that (18) can be obtained from (36) and (39).

Indeed, from (31) and (32) one has

$$
d_{n}=0, \quad B_{0}=1, \quad B_{n}\left(d_{1}, d_{2}, \ldots, d_{n}\right)=0, \quad n=1,2, \ldots,
$$

so that, recalling (37), one has:

$$
\sum_{n=0}^{+\infty} \frac{B_{n}\left(d_{1}, d_{2}, \ldots, d_{n}\right)}{n !}=1, \quad \Phi\left(z, t \mid t_{0}\right)=0 .
$$

Hence, from (36) we have $f\left(x, t \mid 0, t_{0}\right)=\delta(x)$, that coincides with the first expression of (18), whereas from (39) we obtain the second formula of (18). Furthermore, by setting $\beta(t)=0$ in (41), we have (20).

Remark 2 (Proportional case). We assume that (21) holds, i.e., $\beta(t)=\xi r(t)$, with $\xi>0$. We prove that (23) can be obtained from (36) and (39).

Indeed, by virtue of (31) and (32) one has

$$
d_{n}=-\xi(n-1) !, \quad B_{0}=1, \quad B_{n}\left(d_{1}, d_{2}, \ldots, d_{n}\right)=(-\xi)_{n}, \quad n=1,2, \ldots
$$

Recalling the binomial series

$$
\sum_{n=0}^{+\infty}\left(\begin{array}{c}
n+b-1 \\
n
\end{array}\right) x^{n}=\sum_{n=0}^{+\infty}(b)_{n} \frac{x^{n}}{n !}=(1-x)^{-b},
$$


it follows:

$$
\sum_{n=0}^{+\infty} \frac{B_{n}\left(d_{1}, d_{2}, \ldots, d_{n}\right)}{n !}=\sum_{n=0}^{+\infty} \frac{(-\xi)_{n}}{n !}=0, \quad \xi>0 .
$$

Furthermore, since (cf. Erdèlyi et al. [39], p. 213, no. 16)

$$
\sum_{n=0}^{+\infty} \frac{(-\xi)_{n}}{n !} L_{n}(y)=\frac{y^{\xi}}{\Gamma(\xi+1)}, \quad y>0, \xi \geq 0,
$$

from (37) one has:

$$
\begin{gathered}
\Phi\left(z, t \mid t_{0}\right)=\sum_{n=1}^{+\infty} \frac{(-\xi)_{n}}{n !} \frac{d}{d z} L_{n}\left[\frac{z e^{-A\left(t \mid t_{0}\right)}}{R\left(t \mid t_{0}\right)}\right]=\frac{d}{d z} \sum_{n=0}^{+\infty} \frac{(-\xi)_{n}}{n !} L_{n}\left[\frac{z e^{-A\left(t \mid t_{0}\right)}}{R\left(t \mid t_{0}\right)}\right] \\
=\frac{z^{\tau-1}}{\Gamma(\xi)}\left[\frac{e^{-A\left(t \mid t_{0}\right)}}{R\left(t \mid t_{0}\right)}\right]^{\xi} .
\end{gathered}
$$

Hence, making use of (42) and (43) in (36), the first expression of (23) follows. Moreover, from (39), by virtue of (42) and (43), it follows:

$$
\begin{aligned}
& f\left(x, t \mid x_{0}, t_{0}\right)=\frac{1}{\Gamma(\xi)} \exp \left\{-\frac{x_{0}+x e^{-A\left(t \mid t_{0}\right)}}{R\left(t \mid t_{0}\right)}\right\}\left[\frac{e^{-A\left(t \mid t_{0}\right)}}{R\left(t \mid t_{0}\right)}\right]^{\xi} \\
& \quad \times\left\{x^{\xi-1}+\frac{1}{R\left(t \mid t_{0}\right)} \int_{0}^{x} z^{\xi-1} \sqrt{\frac{x_{0} e^{-A\left(t \mid t_{0}\right)}}{x-z}} I_{1}\left[\frac{2 \sqrt{(x-z) x_{0} e^{-A\left(t \mid t_{0}\right)}}}{R\left(t \mid t_{0}\right)}\right] d z\right\}
\end{aligned}
$$

that leads to the second expression of (23), being

$$
\int_{0}^{x}(x-z)^{\xi-1} z^{-1 / 2} I_{1}(2 a \sqrt{z}) d z=-\frac{x^{\xi-1}}{a}+\Gamma(\xi) a^{-\xi} x^{(\xi-1) / 2} I_{\tilde{\zeta}-1}(2 a \sqrt{x}), \quad \operatorname{Re} a \geq 0 .
$$

Finally, recalling that

$$
\sum_{n=1}^{k}(-1)^{n}\left(\begin{array}{l}
k-1 \\
n-1
\end{array}\right) \frac{(-\xi)_{n}}{n !}=\frac{(\xi)_{k}}{k !}, \quad k=1,2 \ldots
$$

from (41) one obtains (26).

\section{Periodic Intensity Functions}

Periodic immigration and periodic growth intensity functions play an important role in the description of the evolution of dynamic systems influenced by seasonal immigration or other regular environmental cycles. Furthermore, the population dynamics can be affected by noise of periodic intensity. Therefore, in this section we assume that the growth intensity function $\alpha(t)$, or the immigration intensity function $\beta(t)$ or the noise intensity $r(t)$ have some kind of periodicity (cf. for instance, Coleman et al. [40], Keeling and Rohani [41]).

\subsection{Periodic Immigration Intensity Function}

We consider the time-inhomogeneous Feller process $X(t)$ such that

$$
A_{1}(x, t)=\alpha x+\xi r(t), \quad A_{2}(x, t)=2 r(t) x,
$$


with $\alpha \in \mathbb{R}, \xi>0$ and a zero-flux condition in the zero-state. We assume that $r(t)$ is a periodic function of period $Q_{1}$. From (15) for $n=0,1, \ldots$ one has $A\left(t+n Q_{1} \mid t_{0}\right)=$ $\alpha\left(t+n Q_{1}-t_{0}\right)$ and

$$
\begin{aligned}
& R\left(t+n Q_{1} \mid t_{0}\right)=\int_{t_{0}}^{t+n Q_{1}} r(\tau) e^{-A\left(\tau \mid t_{0}\right)} d \tau \\
& \quad=e^{\alpha t_{0}}\left\{\frac{1-e^{-n \alpha Q_{1}}}{1-e^{-\alpha Q_{1}}} \int_{t_{0}}^{t_{0}+Q_{1}} r(\tau) e^{-\alpha \tau} d \tau+e^{-n \alpha Q_{1}} \int_{t_{0}}^{t} r(\tau) e^{-\alpha \tau} d \tau\right\} .
\end{aligned}
$$

If $\alpha<0$, the process $X(t)$ admits an asymptotic behavior. In this case, from (23), one has:

$$
W(x, t)=\lim _{n \rightarrow+\infty} f\left(x, t+n Q_{1} \mid x_{0}, t_{0}\right)=\frac{1}{x \Gamma(\xi)}\left[\psi_{1}(t) x\right]^{\xi} e^{-\psi_{1}(t) x}, \quad x \geq 0,
$$

where

$$
\psi_{1}(t)=\lim _{n \rightarrow+\infty} \frac{e^{-A\left(t+n Q_{1} \mid t_{0}\right)}}{R\left(t+n Q_{1} \mid t_{0}\right)}=\frac{e^{|\alpha| Q_{1}}-1}{R\left(t+Q_{1} \mid t\right)}, \quad \alpha<0 .
$$

We note that (45) is a gamma density of parameters $\xi$ and $\left[\psi_{1}(t)\right]^{-1}$ for all $t \geq 0$, so that for $\alpha<0$ it follows:

$$
M_{k}(t)=\lim _{n \rightarrow+\infty} \mathrm{E}\left\{\left[X\left(t+n Q_{1}\right)\right]^{k} \mid X\left(t_{0}\right)=x_{0}\right\}=\left[\psi_{1}(t)\right]^{-k}(\xi)_{k}, \quad k=1,2, \ldots,
$$

with $\psi_{1}(t)$ given in (46).

Example 1. The dynamic of a population influenced by seasonal immigration and regular environmental cycles, can be described by the time-inhomogeneous Feller process (44), with

$$
r(t)=v\left[1+c \sin \left(\frac{2 \pi t}{Q_{1}}\right)\right], \quad t \geq 0,
$$

where $v>0$ is the average of the periodic function $r(t)$ of period $Q_{1}, c$ is the amplitude of the oscillations, with $0 \leq c<1$. These choices of parameters ensure that the both the immigration intensity function and the environment noise are positive functions. From (15), for $t \geq t_{0}$ one has $A\left(t \mid t_{0}\right)=\alpha\left(t-t_{0}\right)$ and

$$
R\left(t \mid t_{0}\right)= \begin{cases}v\left(t-t_{0}\right)+\frac{c v Q_{1}}{2 \pi}\left[\cos \left(\frac{2 \pi t_{0}}{Q_{1}}\right)-\cos \left(\frac{2 \pi t}{Q_{1}}\right)\right], & \alpha=0, \\ \frac{v}{\alpha}\left(1-e^{-\alpha\left(t-t_{0}\right)}\right)+\frac{c v Q_{1}}{4 \pi^{2}+Q_{1}^{2} \alpha^{2}}\left\{2 \pi \cos \left(\frac{2 \pi t_{0}}{Q_{1}}\right)\right. & \\ \left.+\alpha Q_{1} \sin \left(\frac{2 \pi t_{0}}{Q_{1}}\right)-e^{-\alpha\left(t-t_{0}\right)}\left[2 \pi \cos \left(\frac{2 \pi t}{Q_{1}}\right)+\alpha Q_{1} \sin \left(\frac{2 \pi t}{Q_{1}}\right)\right]\right\}, & \alpha \neq 0 .\end{cases}
$$

For $\alpha<0$, the asymptotic density and the asymptotic moments are given in (45) and (47), respectively, with

$$
\psi_{1}(t)=\frac{1}{v}\left\{\frac{1}{|\alpha|}-\frac{c Q_{1}}{4 \pi^{2}+Q_{1}^{2} \alpha^{2}}\left[2 \pi \cos \left(\frac{2 \pi t}{Q_{1}}\right)+\alpha Q_{1} \sin \left(\frac{2 \pi t}{Q_{1}}\right)\right]\right\}^{-1} .
$$

In Figures 1-5, we consider the process (44); we assume that $r(t)=0.5[1+0.9 \sin (\pi t)]$ and that at the initial time $t_{0}=0$ the size of population is $X\left(t_{0}\right)=x_{0}=5$. We recall that $\alpha<0(\alpha>0)$ means that the birth intensity of the population is less (greater) than the death intensity. In Figures 1 and 2 we assume that $\alpha=-0.05$, so that the process admits an asymptotic behavior. Specifically, in Figure 1 , for $\xi=1.5$ the transition $p d f$ of $X(t)$ is plotted as function of $x$ on the left and as function of t on the right; the dotted functions indicate the corresponding asymptotic densities given in (45). Furthermore, in Figure 2, the conditional mean and variance of the population size and the related 
asymptotic behaviors are shown as function of $t$ for various choices of $\xi$. In Figures $3-5$ we assume that $\alpha=0.05$ (on the left) and $\alpha=0$ (on the right), so that $X(t)$ does not admit an asymptotic behavior being $\alpha \geq 0$. In particular, in Figures 3, for $\xi=0.6$ the transition densities of the process (44) are plotted as function of $x$. Finally, in Figures 4 and 5 , the conditional mean and variance are plotted as function of $t$ for various choices of $\xi$; we note that the mean and the variance of the population size don't have an upper bound as tincreases.

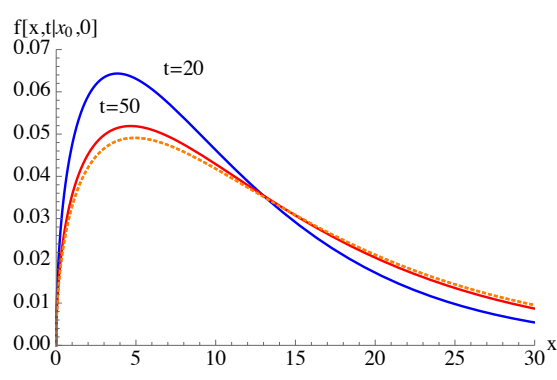

(a) Transition densities as function of $x$

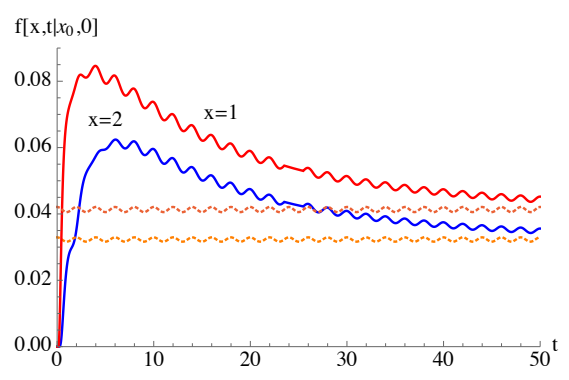

(b) Transition densities as function of $t$

Figure 1. For the process (44), with $x_{0}=5, \alpha=-0.05, \xi=1.5$ and $r(t)=0.5[1+0.9 \sin (\pi t)]$, the transition densities are plotted. The dotted functions indicate the corresponding asymptotic densities.

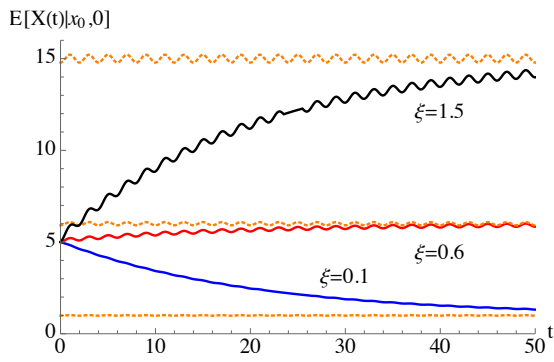

(a) Conditional means

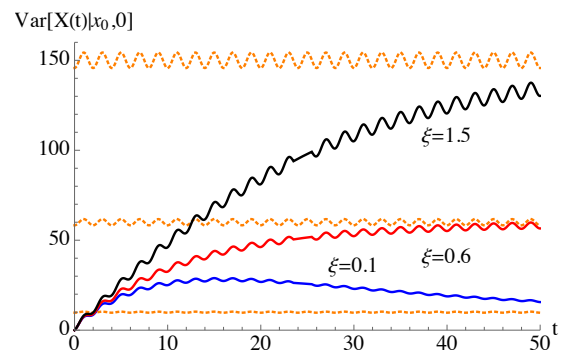

(b) Conditional variances

Figure 2. For the process of Figure 1, the conditional means and variances are plotted as function of $t$ for various choices of $\xi$. The dotted functions indicate the corresponding asymptotic means and variances.

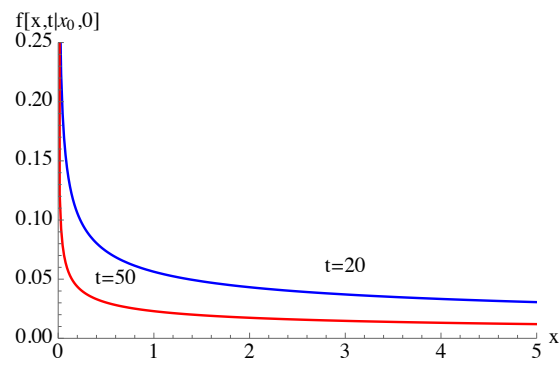

(a) Transition densities for $\alpha=0.05$

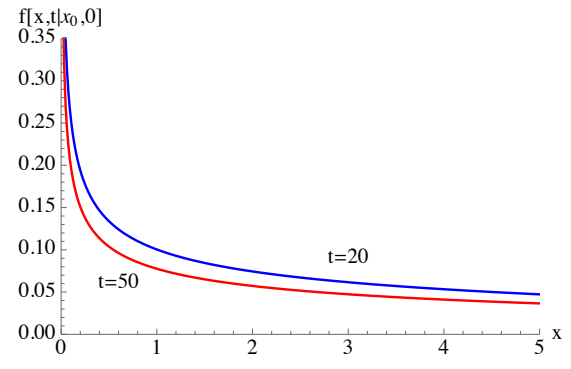

(b) Transition densities for $\alpha=0$

Figure 3. For the process (44), with $x_{0}=5, \xi=0.6$ and $r(t)=0.5[1+0.9 \sin (\pi t)]$, the transition densities are plotted as function of $x$. 


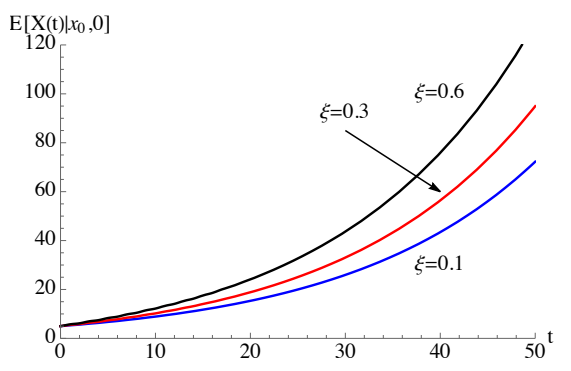

(a) Conditional means for $\alpha=0.05$

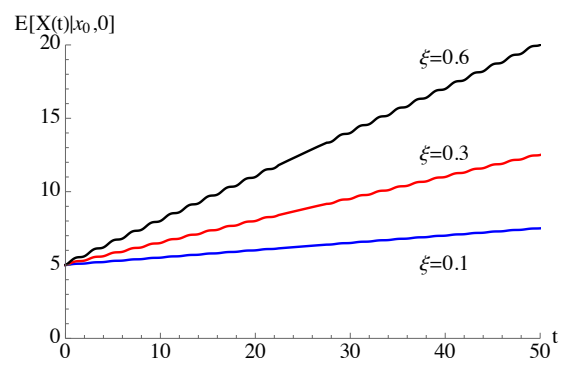

(b) Conditional means for $\alpha=0$

Figure 4. For the process of Figure 3, the conditional means are plotted as function of $t$ for various choices of $\xi$.

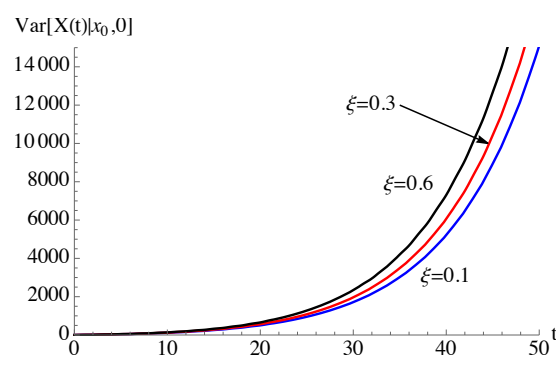

(a) Conditional variances for $\alpha=0.05$

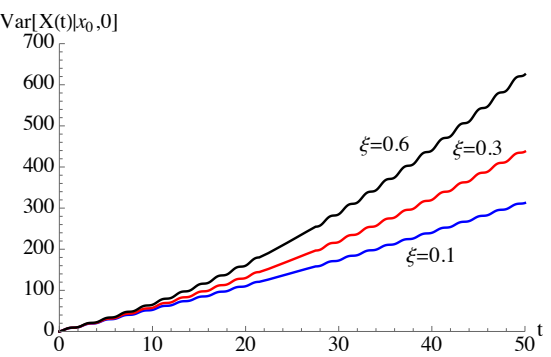

(b) Conditional variances for $\alpha=0$

Figure 5. For the process of Figure 3, the conditional variances are plotted as function of $t$ for various choices of $\xi$.

\subsection{Periodic Growth Intensity Function}

We consider the time-inhomogeneous Feller process $X(t)$ such that

$$
A_{1}(x, t)=\alpha(t) x+\xi r, \quad A_{2}(x)=2 r x
$$

with $r>0, \xi>0$ and a zero-flux condition in the zero-state. We assume that $\alpha(t)$ is a periodic function of period $Q_{2}$ and let

$$
\bar{\alpha}=\frac{A\left(t+Q_{2} \mid t\right)}{Q_{2}}=\frac{1}{Q_{2}} \int_{t}^{t+Q_{2}} \alpha(\tau) d \tau
$$

be the mean of $\alpha(t)$ in the period $Q_{2}$. From (15), for $n=0,1, \ldots$ one has:

$$
\begin{aligned}
& A\left(t+n Q_{2} \mid t_{0}\right)=\int_{t_{0}}^{t+n Q_{2}} \alpha(\tau) d \tau=n A\left(t_{0}+Q_{2} \mid t_{0}\right)+A\left(t \mid t_{0}\right)=n \bar{\alpha} Q_{2}+A\left(t \mid t_{0}\right), \\
& R\left(t+n Q_{2} \mid t_{0}\right)=r \int_{t_{0}}^{t+n Q_{2}} e^{-A\left(\tau \mid t_{0}\right)} d \tau \\
& =r\left\{\frac{1-e^{-n \bar{\alpha} Q_{2}}}{1-e^{\bar{\alpha} Q_{2}}} \int_{t_{0}}^{t_{0}+Q_{2}} e^{-A\left(\tau \mid t_{0}\right)} d \tau+e^{-n \bar{\alpha} Q_{2}} \int_{t_{0}}^{t} e^{-A\left(\tau \mid t_{0}\right)} d \tau\right\} .
\end{aligned}
$$

If $\bar{\alpha}<0$, the process $X(t)$ admits an asymptotic behavior and, from (23), one has:

$$
W(x, t)=\lim _{n \rightarrow+\infty} f\left(x, t+n Q_{2} \mid x_{0}, t_{0}\right)=\frac{1}{x \Gamma(\xi)}\left[\psi_{2}(t) x\right]^{\xi} e^{-\psi_{2}(t) x}, \quad x \geq 0,
$$

where

$$
\psi_{2}(t)=\lim _{n \rightarrow+\infty} \frac{e^{-A\left(t+n Q_{2} \mid t_{0}\right)}}{R\left(t+n Q_{2} \mid t_{0}\right)}=\frac{e^{|\bar{\alpha}| Q_{2}}-1}{R\left(t+Q_{2} \mid t\right)}, \quad \bar{\alpha}<0 .
$$


We note again that (51) is a gamma density of parameters $\xi$ and $\left[\psi_{2}(t)\right]^{-1}$ for all $t \geq 0$; hence, for $\bar{\alpha}<0$ we have:

$$
M_{k}(t)=\lim _{n \rightarrow+\infty} \mathrm{E}\left\{\left[X\left(t+n Q_{2}\right)\right]^{k} \mid X\left(t_{0}\right)=x_{0}\right\}=\left[\psi_{2}(t)\right]^{-k}(\xi)_{k}, \quad k=1,2, \ldots,
$$

with $\psi_{2}(t)$ given in (52).

Example 2. For the time-inhomogeneous Feller process (49), we consider the flexible growth intensity function

$$
\alpha(t)=\eta-\frac{2 \pi b}{Q_{2}} \frac{\cos \left(\frac{2 \pi t}{Q_{2}}\right)}{1+b \sin \left(\frac{2 \pi t}{Q_{2}}\right)}, \quad t \geq 0,
$$

where $\eta \in \mathbb{R}, Q_{2}$ is the period of $\alpha(t)$ and $b$ determines the amplitude of the oscillations, with $0 \leq b<1$. As shown in Figure 6, the growth intensity function (54) can be positive, negative or zero at different time instants; furthermore, different choices of the parameter $b$ make the function (54) less or more asymmetric in a period. Hence, the variety of shapes exhibits by $\alpha(t)$ allows to model several population growth trends.

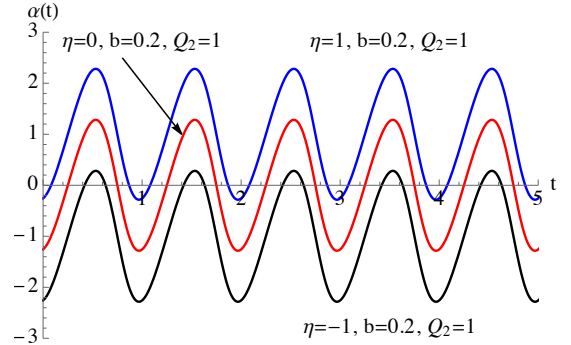

(a) $b=0.2$

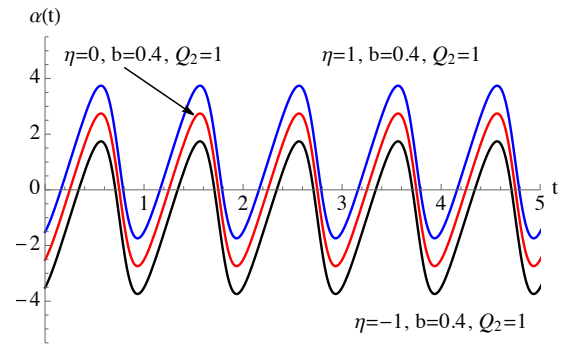

(b) $b=0.4$

Figure 6. The growth intensity function $\alpha(t)$, given in (54), is plotted as function of $t$ for some choices of the parameters.

From (15), making use of (54), for $t \geq t_{0}$ one obtains the following cumulative growth intensity function

$$
A\left(t \mid t_{0}\right)=\eta\left(t-t_{0}\right)-\ln \left[1+b \sin \left(\frac{2 \pi t}{Q_{2}}\right)\right]+\ln \left[1+b \sin \left(\frac{2 \pi t_{0}}{Q_{2}}\right)\right]
$$

and hence

$$
R\left(t \mid t_{0}\right)= \begin{cases}\frac{r}{1+b \sin \left(\frac{2 \pi t_{0}}{Q_{2}}\right)}\left\{t-t_{0}-\frac{b Q_{2}}{2 \pi}\left[\cos \left(\frac{2 \pi t}{Q_{2}}\right)-\cos \left(\frac{2 \pi t_{0}}{Q_{2}}\right)\right]\right\}, & \eta=0, \\ \frac{r}{1+b \sin \left(\frac{2 \pi t_{0}}{Q_{2}}\right)}\left\{\frac{1-e^{-\eta\left(t-t_{0}\right)}}{\eta}-\frac{2 \pi b Q_{2}}{4 \pi^{2}+Q_{2}^{2} \eta^{2}}\left[e^{-\eta\left(t-t_{0}\right)} \cos \left(\frac{2 \pi t}{Q_{2}}\right)\right.\right. & \\ \left.+\frac{Q_{2} \eta}{2 \pi} e^{-\eta\left(t-t_{0}\right)} \sin \left(\frac{2 \pi t}{Q_{2}}\right)-\cos \left(\frac{2 \pi t_{0}}{Q_{2}}\right)-\frac{Q_{2} \eta}{2 \pi} \sin \left(\frac{2 \pi t_{0}}{Q_{2}}\right)\right\}, & \eta \neq 0 .\end{cases}
$$

From (50) and (54) we have $\bar{\alpha}=\eta$, so that $X(t)$ admits an asymptotic behavior for $\eta<0$; the asymptotic density and the asymptotic moments are given in (51) and (53), respectively, with

$$
\psi_{2}(t)=\frac{1}{r}\left[1+b \sin \left(\frac{2 \pi t}{Q_{2}}\right)\right]\left\{\frac{1}{|\eta|}-\frac{2 \pi b Q_{2}}{4 \pi^{2}+Q_{2}^{2} \eta^{2}}\left[\cos \left(\frac{2 \pi t}{Q_{2}}\right)+\frac{Q_{2} \eta}{2 \pi} \sin \left(\frac{2 \pi t}{Q_{2}}\right)\right]\right\}^{-1} .
$$

In Figures 7-11, we consider the process (49) with $r=1$ and $\alpha(t)=\eta-0.4 \pi \cos (2 \pi t)[1+$ $0.2 \sin (2 \pi t)]^{-1}$; furthermore, we assume that at the initial time $t_{0}=0$ the size of population is $X\left(t_{0}\right)=x_{0}=5$. We note that $\eta<0(\eta>0)$ indicates that the average of birth intensity 
of the population is less (greater) than the average of the death intensity. In Figures 7 and 8 we assume that $\eta=-1$, so that the process admits an asymptotic behavior. In particular, in Figure 7 , for $\xi=1.5$ the transition $p d f$ of $X(t)$ is plotted as function of $x$ on the left and as function of $t$ on the right; the dotted functions indicate the corresponding asymptotic densities, given in (51). Furthermore, in Figure 8 the conditional mean and variance and the related asymptotic behaviors are plotted as function of $t$ for various choices of $\xi$. Instead, in Figures 9-11 we assume that $\eta=1$ on the left and $\eta=0$ on the right. Specifically, in Figure 9, the transition densities of the process (49), for $\xi=1.5$ are shown as function of $x$. Finally, in Figures 10 and 11 the conditional mean and variance of the population size are plotted as function of $t$ for various choices of $\xi$.

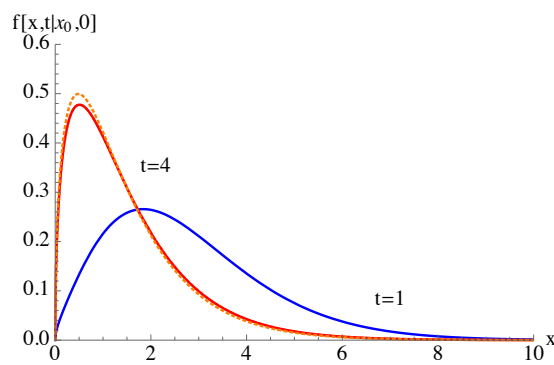

(a) Transition densities as function of $x$

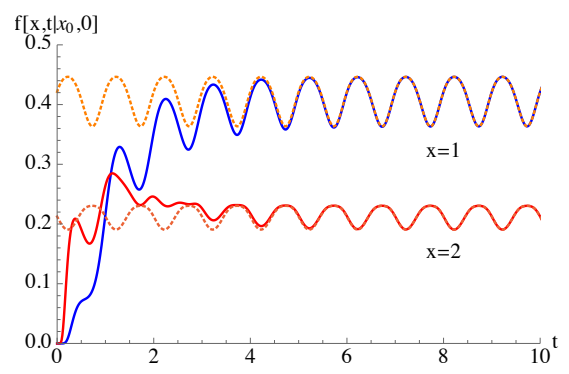

(b) Transition densities as function of $t$

Figure 7. For the process (49), with $x_{0}=5, r=1, \xi=1.5, \eta=-1$ and $\alpha(t)=\eta-0.4 \pi \cos (2 \pi t)[1+$ $0.2 \sin (2 \pi t)]^{-1}$, the transition densities are plotted. The dotted functions indicate the corresponding asymptotic densities.

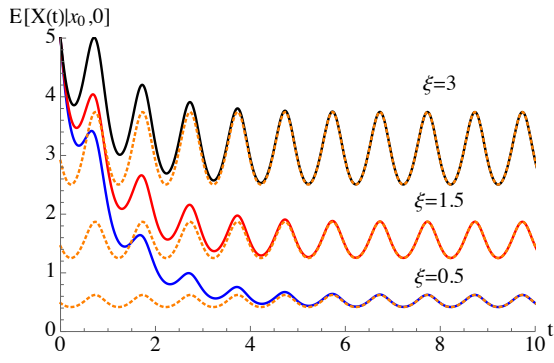

(a) Conditional means

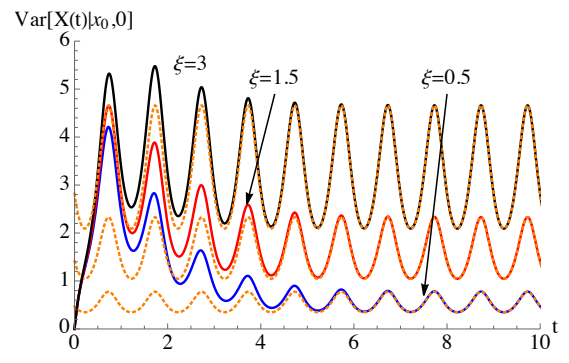

(b) Conditional variances

Figure 8. For the process of Figure 7, the conditional means and variances are plotted as function of $t$ for various choices of $\xi$. The dotted functions indicate the related asymptotic means and variances.

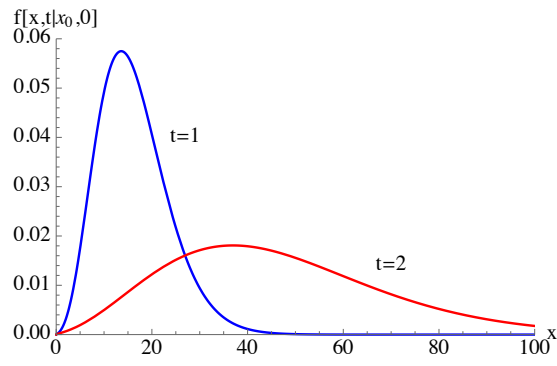

(a) Transition densities for $\eta=1$

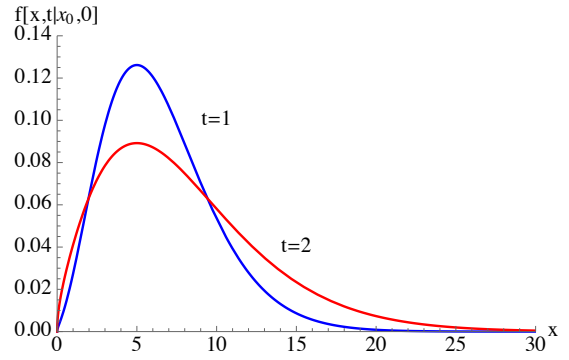

(b) Transition densities for $\eta=0$

Figure 9. For the process of Figure 7, the transition densities are plotted as function of $x$. 


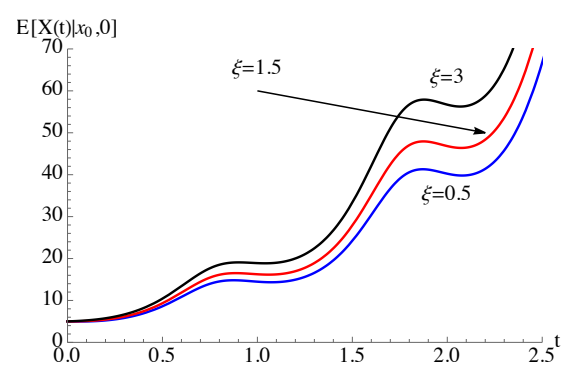

(a) Conditional means for $\eta=1$

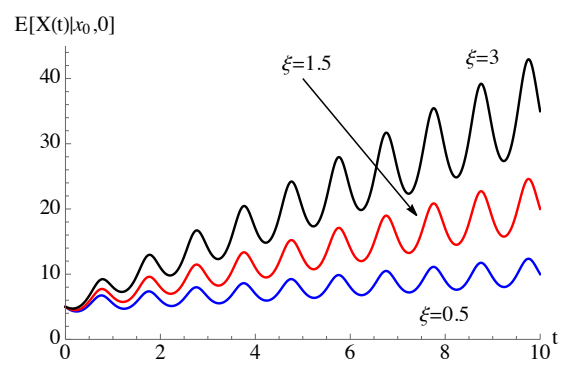

(b) Conditional means for $\eta=0$

Figure 10. For the process of Figure 7 , the conditional means are plotted as function of $t$ for various choices of $\xi$.

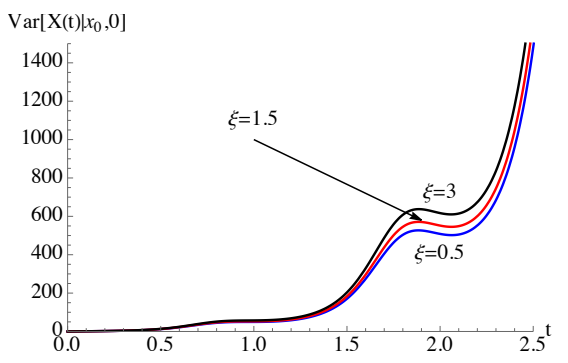

(a) Conditional variances for $\eta=1$

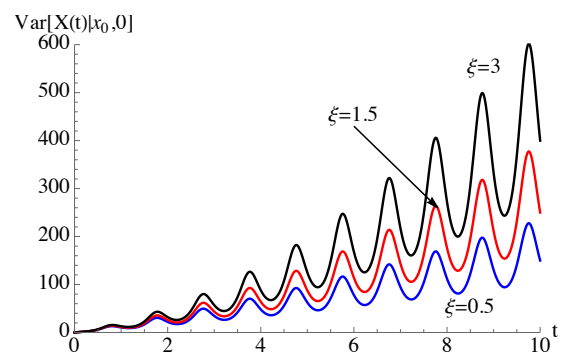

(b) Conditional variances for $\eta=0$

Figure 11. For the process of Figure 7, the conditional variances are plotted as function of $t$ for various choices of $\xi$.

\subsection{Periodic Immigration and Growth Intensity Functions}

We consider the process $X(t)$ such that

$$
A_{1}(x, t)=\alpha(t) x+\xi r(t), \quad A_{2}(x, t)=2 r(t) x,
$$

with $\xi>0$ and a zero-flux condition in the zero-state. We assume that $r(t)$ and $\alpha(t)$ are periodic functions of periods $Q_{1}$ and $Q_{2}$, respectively. We denote by $Q=\operatorname{LCM}\left(Q_{1}, Q_{2}\right)$ the least common multiple between $Q_{1}$ and $Q_{2}$ and let

$$
\widehat{\alpha}=\frac{A(t+Q \mid t)}{Q}=\frac{1}{Q} \int_{t}^{t+Q} \alpha(\tau) d \tau=\frac{1}{Q_{2}} \int_{t}^{t+Q_{2}} \alpha(\tau) d \tau=\bar{\alpha}
$$

be the mean of $\alpha(t)$ in $Q$. From (15) for $n=0,1, \ldots$ one has:

$$
\begin{gathered}
A\left(t+n Q \mid t_{0}\right)=\int_{t_{0}}^{t+n Q} \alpha(\tau) d \tau=n A\left(t_{0}+Q \mid t_{0}\right)+A\left(t \mid t_{0}\right)=n \widehat{\alpha} Q+A\left(t \mid t_{0}\right), \\
R\left(t+n Q \mid t_{0}\right)=\int_{t_{0}}^{t+n Q} r(\tau) e^{-A\left(\tau \mid t_{0}\right)} d \tau \\
=\frac{1-e^{-n \widehat{\alpha} Q}}{1-e^{-\widehat{\alpha} Q}} \int_{t_{0}}^{t_{0}+Q} r(\tau) e^{-A\left(\tau \mid t_{0}\right)} d \tau+e^{-n \widehat{\alpha} Q} \int_{t_{0}}^{t} r(\tau) e^{-A\left(\tau \mid t_{0}\right)} d \tau .
\end{gathered}
$$

If $\widehat{\alpha}<0$, the process $X(t)$ admits an asymptotic behavior and, from (23), one obtains the gamma density:

$$
W(x, t)=\lim _{n \rightarrow+\infty} f\left(x, t+n Q \mid x_{0}, t_{0}\right)=\frac{1}{x \Gamma(\xi)}\left[\psi_{3}(t) x\right]^{\xi} e^{-\psi_{3}(t) x}, \quad x \geq 0
$$

where

$$
\psi_{3}(t)=\lim _{n \rightarrow+\infty} \frac{e^{-A\left(t+n Q \mid t_{0}\right)}}{R\left(t+n Q \mid t_{0}\right)}=\frac{e^{|\widehat{\alpha}| Q}-1}{R(t+Q \mid t)}, \quad \widehat{\alpha}<0 .
$$


Hence, for $\widehat{\alpha}<0$ we have:

$$
M_{k}(t)=\lim _{n \rightarrow+\infty} \mathrm{E}\left\{[X(t+n Q)]^{k} \mid X\left(t_{0}\right)=x_{0}\right\}=\left[\psi_{3}(t)\right]^{-k}(\xi)_{k}, \quad k=1,2, \ldots,
$$

with $\psi_{3}(t)$ given in (59).

Example 3. We consider a population described by the time-inhomogeneous Feller process (57) and we assume that the noise intensity $r(t)$ is defined in (48) and the growth intensity function $\alpha(t)$ is chosen as in (54). In this case, the cumulative growth intensity function $A\left(t \mid t_{0}\right)$ is given in (55) and

$$
R\left(t \mid t_{0}\right)=\frac{v}{1+b \sin \left(\frac{2 \pi t_{0}}{Q_{2}}\right)} \int_{t_{0}}^{t} e^{-\eta\left(\tau-t_{0}\right)}\left[1+c \sin \left(\frac{2 \pi \tau}{Q_{1}}\right)\right]\left[1+b \sin \left(\frac{2 \pi \tau}{Q_{2}}\right)\right] d \tau .
$$

From (50) and (54) we have $\widehat{\alpha}=\eta$. For $\eta<0$, the asymptotic density and the asymptotic moments are given in (58) and (60), respectively, with

$$
\psi_{3}(t)=\frac{1}{v}\left[1+b \sin \left(\frac{2 \pi t}{Q_{2}}\right)\right]\left\{\frac{1}{|\eta|}-c Q_{1} B_{1}(t)-b Q_{2} B_{2}(t)-\frac{b c Q_{1} Q_{2}}{2}[C(t)-D(t)]\right\}^{-1},
$$

where

$$
\begin{aligned}
& B_{i}(t)=\frac{2 \pi \cos \left(\frac{2 \pi t}{Q_{i}}\right)+Q_{i} \eta \sin \left(\frac{2 \pi t}{Q_{i}}\right)}{4 \pi^{2}+Q_{i}^{2} \eta^{2}}, \quad i=1,2, \\
& C(t)=\frac{Q_{1} Q_{2} \eta \cos \left(2 \pi \frac{Q_{1}-Q_{2}}{Q_{1} Q_{2}} t\right)-2 \pi\left(Q_{1}-Q_{2}\right) \sin \left(2 \pi \frac{Q_{1}-Q_{2}}{Q_{1} Q_{2}} t\right)}{4 \pi^{2}\left(Q_{1}-Q_{2}\right)^{2}+Q_{1}^{2} Q_{2}^{2} \eta^{2}}, \\
& D(t)=\frac{Q_{1} Q_{2} \eta \cos \left(2 \pi \frac{Q_{1}+Q_{2}}{Q_{1} Q_{2}} t\right)-2 \pi\left(Q_{1}+Q_{2}\right) \sin \left(2 \pi \frac{Q_{1}+Q_{2}}{Q_{1} Q_{2}} t\right)}{4 \pi^{2}\left(Q_{1}+Q_{2}\right)^{2}+Q_{1}^{2} Q_{2}^{2} \eta^{2}} .
\end{aligned}
$$

In Figures 12-16, we assume that the noise intensity function is $r(t)=0.5[1+0.9 \sin (\pi t)]$ and the growth intensity function is $\alpha(t)=\eta-0.4 \pi \cos (2 \pi t)[1+0.2 \sin (2 \pi t)]^{-1}$; since $r(t)$ has period $Q_{1}=2$ and $\alpha(t)$ has period $Q_{2}=1$, one has $Q=\operatorname{LCM}\left(Q_{1}, Q_{2}\right)=2$. Furthermore, the size of population at time $t_{0}=0$ is $X(0)=5$. In Figures 12 and 13 we assume that $\eta=-1$, so that the process admits an asymptotic behavior. Specifically, in Figure 12 , for $\xi=1.5$ the transition $p d f$ of the process (57) is plotted as function of $x$ on the left and as function of t on the right; the dotted functions indicate the corresponding asymptotic densities, given in (51). Furthermore, in Figure 13 the conditional mean and variance and the related asymptotic behaviors are plotted as function of $t$ for various choices of $\xi$. Comparing Figures 8 and 13 we can highlight the effect of the periodic noise intensity on the conditional mean and variance of the population size for a fixed periodic growth intensity function. In Figures 14-16, we consider $\eta=1$ on the left and $\eta=0$ on the right; in these cases the process does not exhibit an asymptotic behavior, being $\eta \geq 0$. In Figure 14, for $\xi=1.5$ the transition densities of the process are plotted as function of $x$. Finally, in Figures 15 and 16 the conditional mean and variance of the population size are shown as function of $t$ for various choices of $\xi$. 


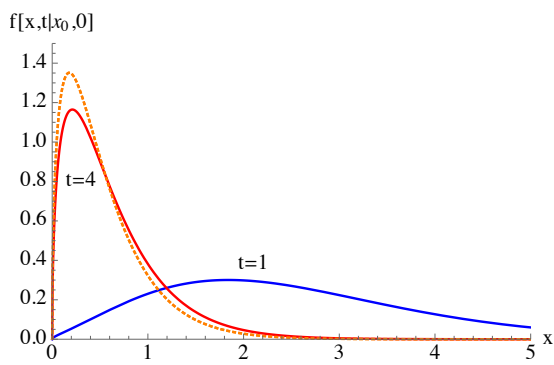

(a) Transition densities as function of $x$

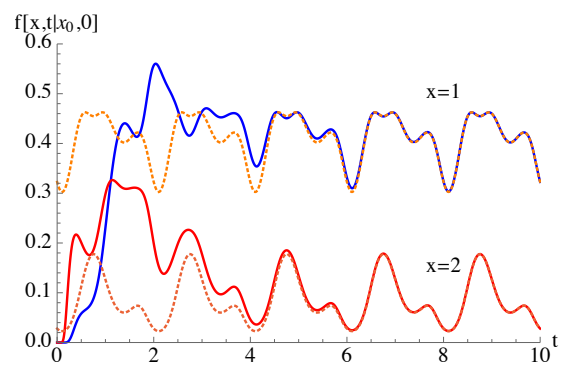

(b) Transition densities as function of $t$

Figure 12. For the process (57), with $x_{0}=5, \xi=1.5, \eta=-1, \alpha(t)=\eta-0.4 \pi \cos (2 \pi t)[1+$ $0.2 \sin (2 \pi t)]^{-1}$ and $r(t)=0.5[1+0.9 \sin (\pi t)]$, the transition densities are plotted. The dotted functions indicate the related asymptotic densities.

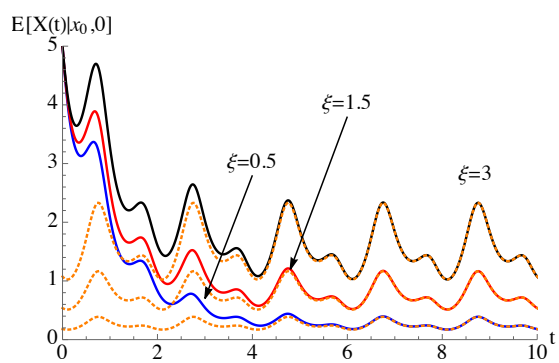

(a) Conditional means

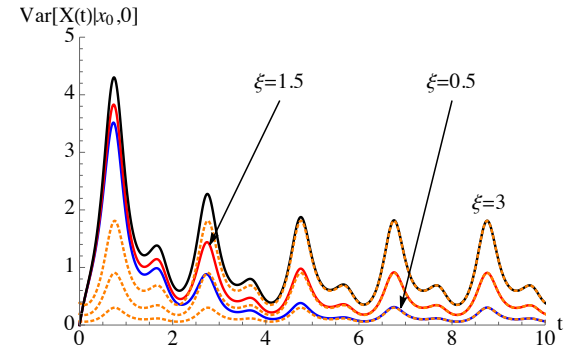

(b) Conditional variances

Figure 13. For the process of Figure 12, the conditional means and the conditional variances are plotted as function of $t$ for various choices of $\xi$. The dotted functions indicate the corresponding asymptotic means and variances.

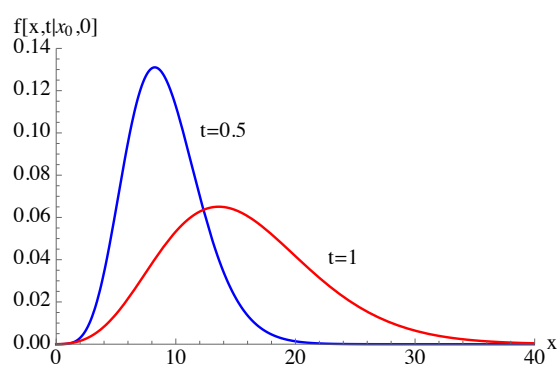

(a) Transition densities for $\eta=1$

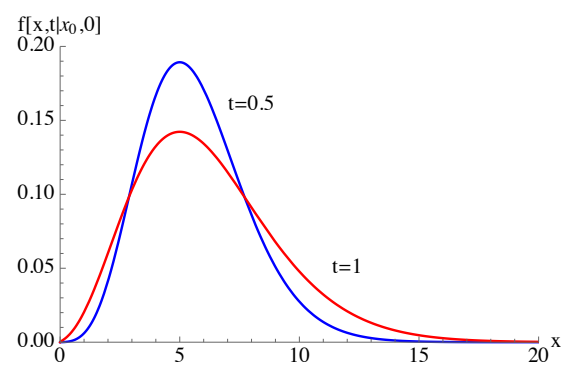

(b) Transition densities for $\eta=0$

Figure 14. For the process of Figure 12, the transition densities are plotted as function of $x$.

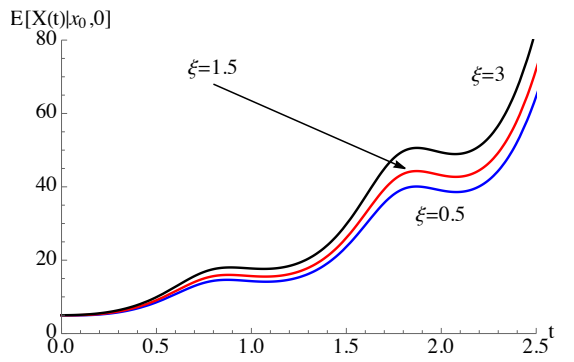

(a) Conditional means for $\eta=1$

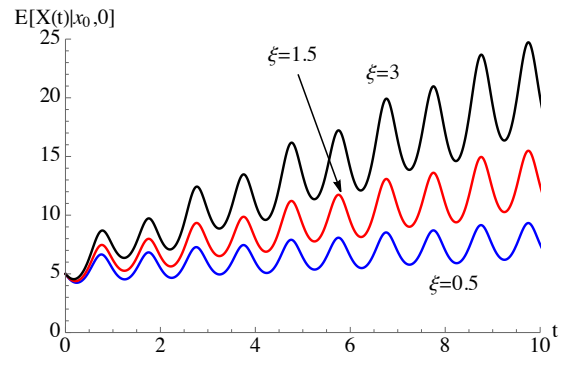

(b) Conditional means for $\eta=0$

Figure 15. For the process of Figure 12, the conditional means are plotted as function of $t$ for various choices of $\xi$. 


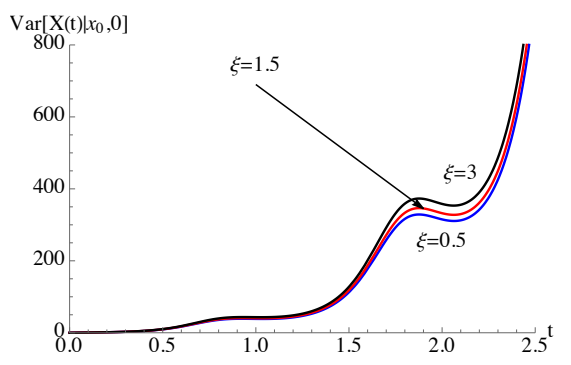

(a) Conditional variances for $\eta=1$

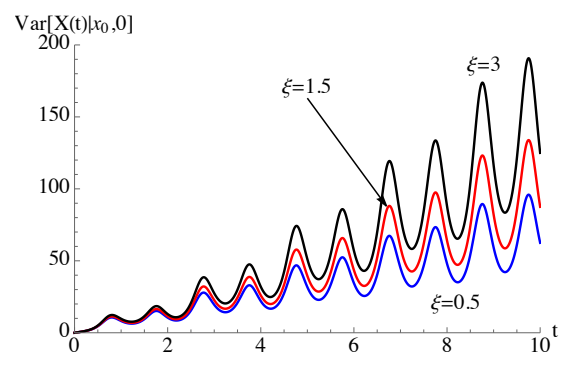

(b) Conditional variances for $\eta=0$

Figure 16. For the process of Figure 12, the conditional variances are plotted as function of $t$ for various choices of $\xi$.

\subsection{Periodic Immigration, Growth and Noise Intensity Functions}

We consider the time-inhomogeneous Feller process $X(t)$ having infinitesimal moments (1), with a zero-flux condition in the zero-state. We assume that $r(t), \alpha(t)$ and $\beta(t)$ are periodic functions of periods $Q_{1}, Q_{2}$ and $Q_{3}$, respectively. Some, but not all, of these functions can be constant. We denote by $Q$ the least common multiple of the periods related to the periodic functions and by $\widehat{\alpha}$ the mean of $\alpha(t)$ in $Q$, so that relations in (58) hold.

If $\widehat{\alpha}<0$, the process $X(t)$ admits an asymptotic behavior. In this case, from (16) one obtains the asymptotic mean and variance:

$$
\begin{aligned}
M_{1}(t) & =\lim _{n \rightarrow+\infty} \mathrm{E}\left\{X(t+n Q) \mid X\left(t_{0}\right)=x_{0}\right\}=\frac{1}{e^{|\widehat{\alpha}| Q}-1} \int_{t}^{t+Q} \beta(u) e^{-A(u \mid t)} d u, \\
V(t)= & \lim _{n \rightarrow+\infty} \operatorname{Var}\left\{X(t+n Q) \mid X\left(t_{0}\right)=x_{0}\right\}=\frac{2}{\left(e^{|\widehat{\alpha}| Q}-1\right)^{2}\left(1+e^{|\widehat{\alpha}| Q)}\right.} \\
& \times \int_{t}^{t+Q} \beta(u) e^{-A(u \mid t)}\left[R(u \mid t)+e^{|\widehat{\alpha}| Q} \int_{u}^{t+Q} r(\tau) e^{-A(\tau \mid t)} d \tau\right] d u .
\end{aligned}
$$

Note that if $\beta(t)=\xi r(t)$, from (62) and (63) one has $M_{1}(t)=\xi\left[\psi_{3}(t)\right]^{-1}$ and $V(t)=$ $\xi\left[\psi_{3}(t)\right]^{-2}$, with $\psi_{3}(t)$ defined in (59).

Example 4. We consider the process (1) and we assume that $\beta(t)=\beta>0, r(t)$ is given in (48) and $\alpha(t)$ is defined in (54). In this case, the immigration rate is constant, whereas the growth and the noise intensity functions are periodic functions with different periods. Expressions (55) and (61) for $A\left(t \mid t_{0}\right)$ and $R\left(t \mid t_{0}\right)$ hold. Furthermore, from (50) and (54) we have $\widehat{\alpha}=\eta$, so that for $\eta<0$ the process admits an asymptotic behavior.

We assume that the noise intensity function $r(t)$ has period $Q_{1}=2$ and the growth intensity function $\alpha(t)$ has period $Q_{2}=1$, so that $Q=\operatorname{LCM}\left(Q_{1}, Q_{2}\right)=2$. In Figure 17, the conditional mean and variance and the related asymptotic behaviors are plotted as function of $t$ for various choices of $\beta$. We note that the conditional mean is not affect to the periodicity of $r(t)$, whereas the conditional variance depends on the different periodicities of the growth intensity function $\alpha(t)$ and of the noise intensity function $r(t)$. 


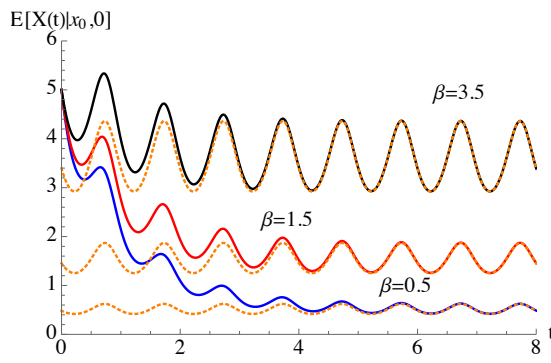

(a) Conditional means

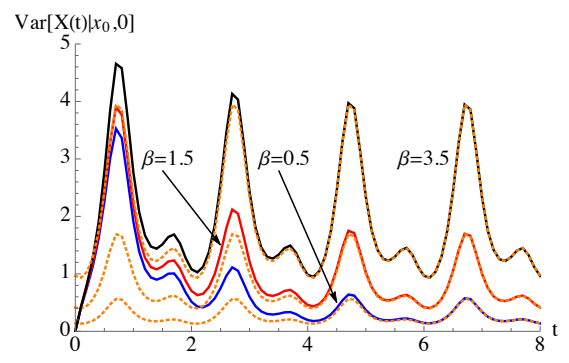

(b) Conditional variances

Figure 17. For the process (1), with $x_{0}=5, \eta=-1, \alpha(t)=\eta-0.4 \pi \cos (2 \pi t)[1+0.2 \sin (2 \pi t)]^{-1}$ and $r(t)=0.5[1+0.9 \sin (\pi t)]$, the conditional means and variances are plotted as function of $t$ for various choices of $\beta$. The dotted functions indicate the corresponding asymptotic means and variances.

Example 5. We consider the process (1) and we assume that $r(t)=r>0, \alpha(t)$ is defined in (54) and

$$
\beta(t)=\beta\left[1+c \sin \left(\frac{2 \pi t}{Q_{1}}\right)\right], \quad t \geq 0,
$$

where $\beta>0$ is the average of the periodic function $\beta(t)$ of period $Q_{1}, c$ is the amplitude of the oscillations, with $0 \leq c<1$. Differently from Example 4, the noise intensity is constant, whereas the growth and the immigration intensity functions are periodic functions with different periods. Expressions (55) and (56) for $A\left(t \mid t_{0}\right)$ and $R\left(t \mid t_{0}\right)$ hold. Furthermore, from (50) and (54) we have $\widehat{\alpha}=\eta$, so that for $\eta<0$ the process admits an asymptotic behavior.

We assume that the immigration intensity function $\beta(t)$, given in (64), has period $Q_{1}=2$ and the growth intensity function $\alpha(t)$ has period $Q_{2}=1$, so that $Q=\operatorname{LCM}\left(Q_{1}, Q_{2}\right)=2$. In Figure 18, the conditional mean and variance and the related asymptotic behaviors are plotted as function of $t$ for various choices of $\beta$. We note that both the mean and the variance depend on the periodicities of the intensity functions $\alpha(t)$ and $\beta(t)$.

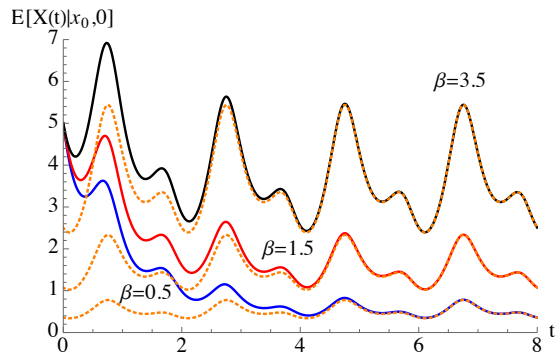

(a) Conditional means

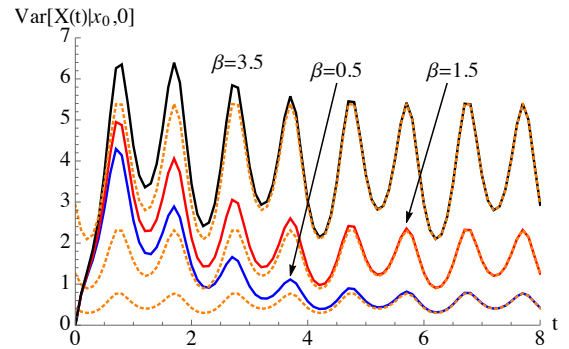

(b) Conditional variances

Figure 18. For the process (1), with $x_{0}=5, \eta=-1, r=1, \alpha(t)=\eta-0.4 \pi \cos (2 \pi t)[1+$ $0.2 \sin (2 \pi t)]^{-1}$ and $\beta(t)=\beta[1+0.9 \sin (\pi t)]$, the conditional means and variances are plotted as function of $t$ for various choices of $\beta$. The dotted functions indicate the related asymptotic means and variances.

\section{Concluding Remarks}

In this paper, we considered a time-inhomogeneous Feller-type diffusion process $\left\{X(t), t \geq t_{0}\right\}, t_{0} \geq 0$, with infinitesimal drift $A_{1}(x, t)=\alpha(t) x+\beta(t)$ and infinitesimal variance $A_{2}(x, t)=2 r(t) x$, defined in the state-space $[0,+\infty)$, with a zero-flux condition in the zero-state. We have assumed that $\alpha(t) \in \mathbb{R}, \beta(t) \geq 0, r(t)>0$ for all $t \geq t_{0}$. This diffusion process plays a relevant role in several biological applications and can be derived as the continuous approximation of the time-inhomogeneous birth-death process with immigration. In the general case, the transition density and the conditional moments are explicitly obtained. Some special situations are analyzed: (i) the absence of immigration with $\beta(t)=0$, (ii) the proportional case in which $\beta(t)=\xi r(t)$, with $\xi>0$, and (iii) the timehomogeneous case. Sometimes in the dynamics of populations it is necessary to consider 
periodic intensity functions. Indeed, periodic immigration and periodic growth intensity functions play an important role in the description of the evolution of dynamic systems influenced by seasonal immigration or other regular environmental cycles. Furthermore, the population dynamics can be affected by noise of periodic intensity. Therefore, we have assumed that the growth intensity function $\alpha(t)$, or the immigration intensity function $\beta(t)$ or the noise intensity $r(t)$ have some kind of periodicity. In these cases, the asymptotic behaviors of the transition pdf and of the moments are discussed. Various numerical computations are performed to analyze how the population dynamics is affected by the periodic intensity functions.

Author Contributions: The authors have participated equally in the development of this work, either in the theoretical and computational aspects. All authors have read and agreed to the published version of the manuscript.

Funding: This research is partially supported by MIUR-PRIN 2017, Project “Stochastic Models for Complex Systems" and by the Ministerio de Economía, Industria y Competitividad, Spain, under Grant MTM2017-85568-P. This research received no external funding.

Institutional Review Board Statement: Not applicable.

Informed Consent Statement: Not applicable.

Acknowledgments: The authors are members of the research group GNCS of INdAM.

Conflicts of Interest: The authors declare no conflict of interest.

\section{Appendix A. Proof of Proposition 1}

We solve (12) with initial condition (13) making use of the method of characteristics (cf. for instance, Williams [42]). We consider the differential equations:

$$
\frac{d t}{d \xi}=1, \quad \frac{d s}{d \xi}=-s[\alpha(t)-s r(t)], \quad \frac{d M}{d \xi}=-s \beta(t) M,
$$

and the initial conditions:

$$
t\left(w, \xi=t_{0}\right)=t_{0}, \quad s\left(w, \xi=t_{0}\right)=w, \quad M\left(w, \xi=t_{0}\right)=e^{-w x_{0}} .
$$

The first expression of (A1), with the first initial condition in (A2), leads to $t=\xi$. Solving the second equation in (A1) with $t=\xi$ and recalling of the second formula of (A2), it follows:

$$
s=\frac{w e^{-A\left(\xi \mid t_{0}\right)}}{1-w R\left(\xi \mid t_{0}\right)}
$$

Then, solving the third equation in (A1), with the related initial condition in (A2) and with $t=\xi$ and $s$ given in (A3), we obtain:

$$
M(w, \xi)=e^{-w x_{0}} \exp \left\{-w \int_{t_{0}}^{\xi} \frac{\beta(u) e^{-A\left(u \mid t_{0}\right)}}{1-w R\left(u \mid t_{0}\right)} d u\right\} .
$$

From (A3) with $\xi=t$, we also have

$$
w=\frac{s e^{A\left(t \mid t_{0}\right)}}{1+s e^{A\left(t \mid t_{0}\right)} R\left(t \mid t_{0}\right)} .
$$

Finally, Equation (14) follows from (A4), recalling that $\xi=t$ and making use of (A5). 


\section{Appendix B. Proof of Proposition 2}

Setting $\beta(t)=0$ in (14), we immediately obtain (17), from which one has:

$$
\begin{aligned}
M^{*}\left(s, t \mid x_{0}, t_{0}\right) & =\int_{0}^{+\infty} e^{-s x} f^{*}\left(x, t \mid x_{0}, t_{0}\right) d x \\
& =\exp \left\{-\frac{x_{0}}{R\left(t \mid t_{0}\right)}\right\} \exp \left\{\frac{x_{0}}{R\left(t \mid t_{0}\right)} \frac{1}{1+s e^{A\left(t \mid t_{0}\right)} R\left(t \mid t_{0}\right)}\right\}, \quad s>0 .
\end{aligned}
$$

Setting

$$
1+s e^{A\left(t \mid t_{0}\right)} R\left(t \mid t_{0}\right)=z, \quad \frac{x e^{-A\left(t \mid t_{0}\right)}}{R\left(t \mid t_{0}\right)}=y,
$$

in (A6), we obtain

$$
\begin{aligned}
\int_{0}^{+\infty} e^{-z y}\left\{e^{y} f^{*}\right. & {\left.\left[R\left(t \mid t_{0}\right) e^{A\left(t \mid t_{0}\right)} y, t \mid x_{0}, t_{0}\right]\right\} d y } \\
= & \frac{e^{-A\left(t \mid t_{0}\right)}}{R\left(t \mid t_{0}\right)} \exp \left\{-\frac{x_{0}}{R\left(t \mid t_{0}\right)}\right\} \exp \left\{\frac{x_{0}}{R\left(t \mid t_{0}\right)} \frac{1}{z}\right\} .
\end{aligned}
$$

Since

$$
\int_{0}^{+\infty} e^{-p y}\left\{\sqrt{\frac{a}{y}} I_{1}(2 \sqrt{a y})+\delta(y)\right\} d y=e^{a / p},
$$

taking the inverse Laplace transforms on both sides of (A8), it follows:

$$
\begin{aligned}
f^{*}\left[R\left(t \mid t_{0}\right) e^{A\left(t \mid t_{0}\right)} y, t \mid x_{0}, t_{0}\right] & =e^{-y} \frac{e^{-A\left(t \mid t_{0}\right)}}{R\left(t \mid t_{0}\right)} \exp \left\{-\frac{x_{0}}{R\left(t \mid t_{0}\right)}\right\} \\
& \times\left\{\delta(y)+\sqrt{\frac{x_{0}}{y R\left(t \mid t_{0}\right)}} I_{1}\left(2 \sqrt{\frac{x_{0} y}{R\left(t \mid t_{0}\right)}}\right)\right\} .
\end{aligned}
$$

Equation (18) follows from (A9), applying again the transformation $x=R\left(t \mid t_{0}\right) e^{A\left(t \mid t_{0}\right)} y$ and recalling that $\delta(a x)=\delta(x) /|a|$.

\section{Appendix C. Proof of Proposition 3}

We note that

$\frac{d}{d u} \ln \left\{1+s e^{A\left(t \mid t_{0}\right)}\left[R\left(t \mid t_{0}\right)-R\left(u \mid t_{0}\right)\right]\right\}=-\frac{s r(u) e^{A(t \mid u)}}{1+s e^{A\left(t \mid t_{0}\right)}\left[R\left(t \mid t_{0}\right)-R\left(u \mid t_{0}\right)\right]}, \quad t_{0} \leq u \leq t$, so that

$$
\begin{aligned}
& \exp \left\{-\int_{t_{0}}^{t} \frac{s \beta(u) e^{A(t \mid u)}}{1+s e^{A\left(t \mid t_{0}\right)}\left[R\left(t \mid t_{0}\right)-R\left(u \mid t_{0}\right)\right]}\right\}=\exp \left\{-\xi \int_{t_{0}}^{t} \frac{s r(u) e^{A(t \mid u)}}{1+s e^{A\left(t \mid t_{0}\right)}\left[R\left(t \mid t_{0}\right)-R\left(u \mid t_{0}\right)\right]}\right\} \\
& =\left[1+s e^{A\left(t \mid t_{0}\right)} R\left(t \mid t_{0}\right)\right]^{-\zeta} .
\end{aligned}
$$

Hence, making use of (A10) in (14), one obtains (22).

To derive (23), we distinguish two cases: (i) $x_{0}=0$ and (ii) $x_{0}>0$.

Case (i) If $x_{0}=0$, the expression (22) becomes:

$$
M\left(s, t \mid 0, t_{0}\right)=\left[\frac{e^{-A\left(t \mid t_{0}\right)}}{R\left(t \mid t_{0}\right)}\right]^{\xi}\left[s+\frac{e^{-A\left(t \mid t_{0}\right)}}{R\left(t \mid t_{0}\right)}\right]^{-\xi}, \quad \xi>0,
$$


which is recognized to be the moment generating function of a gamma distribution of parameters $\xi$ and $e^{-A\left(t \mid t_{0}\right)} / R\left(t \mid t_{0}\right)$ for all $t \geq t_{0}$. Indeed, since (cf. Erdèlyi et al. [43], p. 144, no. 3)

$$
\int_{0}^{+\infty} e^{-s x} x^{v-1} e^{-a x} d x=\Gamma(v)(s+a)^{-v}, \quad \operatorname{Re} v>0,
$$

from (11), for $t \geq t_{0}$ the first formula of (23) immediately follows.

Case (ii) If $x>0$, from (11) and (22) it follows:

$$
\int_{0}^{+\infty} e^{-s x} f\left(x, t \mid x_{0}, t_{0}\right) d x=\left[1+s e^{A\left(t \mid t_{0}\right)} R\left(t \mid t_{0}\right)\right]^{-\xi} \exp \left\{-\frac{s x_{0} e^{A\left(t \mid t_{0}\right)}}{1+s e^{A\left(t \mid t_{0}\right)} R\left(t \mid t_{0}\right)}\right\},
$$

so that, by virtue of (A7), one has:

$$
\begin{gathered}
\int_{0}^{+\infty} e^{-z y}\left\{e^{y} f\left[y R\left(t \mid t_{0}\right) e^{A\left(t \mid t_{0}\right)}, t \mid x_{0}, t_{0}\right]\right\} d y=\frac{e^{-A\left(t \mid t_{0}\right)}}{R\left(t \mid t_{0}\right)} \exp \left\{-\frac{x_{0}}{R\left(t \mid t_{0}\right)}\right\} \\
\times z^{-\xi} \exp \left\{\frac{x_{0}}{z R\left(t \mid t_{0}\right)}\right\}, \quad \xi>0 .
\end{gathered}
$$

Since (cf. Erdèlyi et al. [43], p. 197, no. 18)

$$
\int_{0}^{+\infty} e^{-z y} a^{-v / 2} y^{v / 2} I_{v}(2 \sqrt{a y}) d y=z^{-v-1} e^{a / z}, \quad \operatorname{Re} v>-1,
$$

taking the inverse Laplace transforms on both sides of (A12), for $t \geq t_{0}$ we obtain:

$$
\begin{gathered}
f\left[y R\left(t \mid t_{0}\right) e^{A\left(t \mid t_{0}\right)}, t \mid x_{0}, t_{0}\right]=e^{-y} \frac{e^{-A\left(t \mid t_{0}\right)}}{R\left(t \mid t_{0}\right)} \exp \left\{-\frac{x_{0}}{R\left(t \mid t_{0}\right)}\right\}\left[\frac{x_{0}}{R\left(t \mid t_{0}\right)}\right]^{(1-\xi) / 2} \\
\times y^{(\tilde{\xi}-1) / 2} I_{\tilde{\xi}-1}\left[2 \sqrt{\frac{x_{0} y}{R\left(t \mid t_{0}\right)}}\right], \quad \xi>0 .
\end{gathered}
$$

The second expression of (23) follows from (A13), applying the transformation $x=$ $R\left(t \mid t_{0}\right) e^{A\left(t \mid t_{0}\right)} y$.

\section{Appendix D. Proof of Proposition 4}

We assume that $x \geq 0$ and $t \geq t_{0}$. Recalling (11) and by using (A7) in (30), we have:

$$
\begin{aligned}
\int_{0}^{+\infty} e^{-z y}\left\{e^{y} f\left[y R\left(t \mid t_{0}\right) e^{A\left(t \mid t_{0}\right)}, t \mid 0, t_{0}\right]\right\} d y=\frac{e^{-A\left(t \mid t_{0}\right)}}{R\left(t \mid t_{0}\right)} \\
\times \exp \left\{-(z-1) \int_{t_{0}}^{t} \frac{\beta(u) e^{-A\left(u \mid t_{0}\right)}}{z R\left(t \mid t_{0}\right)-(z-1) R\left(u \mid t_{0}\right)} d u\right\},
\end{aligned}
$$

where

$$
\begin{aligned}
\exp \{ & \left.-(z-1) \int_{t_{0}}^{t} \frac{\beta(u) e^{-A\left(u \mid t_{0}\right)}}{z R\left(t \mid t_{0}\right)-(z-1) R\left(u \mid t_{0}\right)} d u\right\} \\
& =\exp \left\{-\frac{z-1}{z} \int_{t_{0}}^{t} \frac{\beta(u) e^{-A\left(u \mid t_{0}\right)}}{R\left(t \mid t_{0}\right)\left[1-\frac{z-1}{z} \frac{R\left(u \mid t_{0}\right)}{R\left(t \mid t_{0}\right)}\right]} d u\right\} \\
& =\exp \left\{-\sum_{k=1}^{+\infty}\left(1-\frac{1}{z}\right)^{k} \frac{1}{\left[R\left(t \mid t_{0}\right)\right]^{k}} \int_{t_{0}}^{t} \beta(u) e^{-A\left(u \mid t_{0}\right)}\left[R\left(u \mid t_{0}\right)\right]^{k-1} d u\right\} .
\end{aligned}
$$


Note that the last equality in (A14) follows being

$$
0<\frac{z-1}{z} \frac{R\left(u \mid t_{0}\right)}{R\left(t \mid t_{0}\right)}<1, \quad t_{0} \leq u \leq t .
$$

Let $B_{n}\left(d_{1}, d_{2}, \ldots, d_{n}\right)$ be the complete Bell polynomials defined in (31), with $d_{k}$ given in (32). Since (cf. for instance, Comtet [44]):

$$
\exp \left\{\sum_{r=1}^{+\infty} \frac{d_{r}}{r !} \vartheta^{r}\right\}=\sum_{n=0}^{+\infty} \frac{B_{n}\left(d_{1}, d_{2}, \ldots, d_{n}\right)}{n !} \vartheta^{n},
$$

from (A14) one obtains:

$$
\exp \left\{-(z-1) \int_{t_{0}}^{t} \frac{\beta(u) e^{-A\left(u \mid t_{0}\right)}}{z R\left(t \mid t_{0}\right)-(z-1) R\left(u \mid t_{0}\right)} d u\right\}=\sum_{n=0}^{+\infty} \frac{B_{n}\left(d_{1}, d_{2}, \ldots, d_{n}\right)}{n !}\left(1-\frac{1}{z}\right)^{n}
$$

Making use of (A15) in (A14), it follows:

$$
\begin{aligned}
& \int_{0}^{+\infty} e^{-z y}\left\{e^{y} f\left[y R\left(t \mid t_{0}\right) e^{A\left(t \mid t_{0}\right)}, t \mid 0, t_{0}\right]\right\} d y=\frac{e^{-A\left(t \mid t_{0}\right)}}{R\left(t \mid t_{0}\right)}\left\{\sum_{n=0}^{+\infty} \frac{B_{n}\left(d_{1}, d_{2}, \ldots, d_{n}\right)}{n !}\right. \\
& \left.+\sum_{n=1}^{+\infty} \frac{B_{n}\left(d_{1}, d_{2}, \ldots, d_{n}\right)}{n !}\left[\left(1-\frac{1}{z}\right)^{n}-1\right]\right\} .
\end{aligned}
$$

Recalling (34), one obtains

$$
\int_{0}^{+\infty} e^{-z y} \frac{d}{d y} L_{n}(y) d y=\left(1-\frac{1}{z}\right)^{n}-1, \quad \operatorname{Re} z>0,
$$

so that Equation (A16) leads to:

$$
\begin{gathered}
f\left[y R\left(t \mid t_{0}\right) e^{A\left(t \mid t_{0}\right)}, t \mid 0, t_{0}\right]=e^{-y} \frac{e^{-A\left(t \mid t_{0}\right)}}{R\left(t \mid t_{0}\right)}\left\{\sum_{n=0}^{+\infty} \frac{B_{n}\left(d_{1}, d_{2}, \ldots, d_{n}\right)}{n !} \delta(y)\right. \\
\left.+\sum_{n=1}^{+\infty} \frac{B_{n}\left(d_{1}, d_{2}, \ldots, d_{n}\right)}{n !} \frac{d}{d y} L_{n}(y)\right\} .
\end{gathered}
$$

Finally, Equation (36) follows, applying again the transformation $x=y R\left(t \mid t_{0}\right) e^{A\left(t \mid t_{0}\right)}$.

\section{Appendix E. Proof of Proposition 5}

By virtue of (36), for $k=1,2, \ldots$ one has:

$$
\begin{aligned}
\mathrm{E}\left[X^{k}(t) \mid X\left(t_{0}\right)=0\right]=\int_{0}^{+\infty} x^{k} f\left(x, t \mid 0, t_{0}\right) d x \\
\quad=\sum_{n=1}^{+\infty} \frac{B_{n}\left(d_{1}, d_{2}, \ldots, d_{n}\right)}{n !} \int_{0}^{+\infty} x^{k} \exp \left\{-\frac{x e^{-A\left(t \mid t_{0}\right)}}{R\left(t \mid t_{0}\right)}\right\} \frac{d}{d x} L_{n}\left[\frac{x e^{-A\left(t \mid t_{0}\right)}}{R\left(t \mid t_{0}\right)}\right] d x
\end{aligned}
$$

By using the transformation $y=x e^{-A\left(t \mid t_{0}\right)} / R\left(t \mid t_{0}\right)$ on the right-hand side of (A17), one has:

$$
\begin{aligned}
\int_{0}^{+\infty} x^{k} & \exp \left\{-\frac{x e^{-A\left(t \mid t_{0}\right)}}{R\left(t \mid t_{0}\right)}\right\} \frac{d}{d x} L_{n}\left[\frac{x e^{-A\left(t \mid t_{0}\right)}}{R\left(t \mid t_{0}\right)}\right] d x \\
= & {\left[R\left(t \mid t_{0}\right) e^{A\left(t \mid t_{0}\right)}\right]^{k} \int_{0}^{+\infty} y^{k} e^{-y} \frac{d}{d y} L_{n}(y) d y, \quad k=1,2, \ldots }
\end{aligned}
$$


Since

$$
\begin{gathered}
\int_{0}^{+\infty} y^{k} e^{-y} \frac{d}{d y} L_{n}(y) d y=\sum_{r=1}^{n}\left(\begin{array}{l}
n \\
r
\end{array}\right) \frac{(-1)^{r}}{(r-1) !}(k+r-1) ! \\
= \begin{cases}(-1)^{n} k !\left(\begin{array}{c}
k-1 \\
n-1
\end{array}\right), & n=1,2, \ldots, k \\
0, & n=k+1, k+2, \ldots,\end{cases}
\end{gathered}
$$

from (A18) one obtains:

$$
\begin{aligned}
& \int_{0}^{+\infty} x^{k} \exp \left\{-\frac{x e^{-A\left(t \mid t_{0}\right)}}{R\left(t \mid t_{0}\right)}\right\} \frac{d}{d x} L_{n}\left[\frac{x e^{-A\left(t \mid t_{0}\right)}}{R\left(t \mid t_{0}\right)}\right] d x \\
&= \begin{cases}(-1)^{n} k !\left(\begin{array}{c}
k-1 \\
n-1
\end{array}\right)\left[R\left(t \mid t_{0}\right) e^{A\left(t \mid t_{0}\right)}\right]^{k}, & n=0,1, \ldots, k \\
0, & n=k+1, k+2, \ldots\end{cases}
\end{aligned}
$$

Relation (38) follows making use of (A19) in (A17).

\section{Appendix F. Proof of Proposition 7}

Making use of (40), for $k=1,2, \ldots$ it follows:

$$
\begin{aligned}
& \mathrm{E}\left[X^{k}(t) \mid X\left(t_{0}\right)=x_{0}\right]=\int_{0}^{+\infty} x^{k} f\left(x, t \mid x_{0}, t_{0}\right) d x \\
& =\int_{0}^{+\infty} d x x^{k} \int_{0}^{x} f^{*}\left(y, t \mid x_{0}, t_{0}\right) f\left(x-y, t \mid 0, t_{0}\right) d y \\
& =\sum_{i=0}^{k}\left(\begin{array}{c}
k \\
i
\end{array}\right) \int_{0}^{+\infty} y^{i} f^{*}\left(y, t \mid x_{0}, t_{0}\right) d y \int_{0}^{+\infty} z^{k-i} f\left(z, t \mid 0, t_{0}\right) d z .
\end{aligned}
$$

For $k=1$, from (A20) one has

$$
\mathrm{E}\left[X(t) \mid X\left(t_{0}\right)=x_{0}\right]=\int_{0}^{+\infty} y f^{*}\left(y, t \mid x_{0}, t_{0}\right) d y+\int_{0}^{+\infty} z f\left(z, t \mid 0, t_{0}\right) d z,
$$

that leads to the first expression of (16), making use of (20) and (38). Instead, for $k=2,3, \ldots$ from (A20) one obtains:

$$
\begin{aligned}
\mathrm{E}\left[X^{k}(t) \mid X\left(t_{0}\right)=x_{0}\right] & =\int_{0}^{+\infty} z^{k} f\left(z, t \mid 0, t_{0}\right) d z+\int_{0}^{+\infty} y^{k} f^{*}\left(y, t \mid x_{0}, t_{0}\right) d y \\
& +\sum_{i=1}^{k-1}\left(\begin{array}{c}
k \\
i
\end{array}\right) \int_{0}^{+\infty} y^{i} f^{*}\left(y, t \mid x_{0}, t_{0}\right) d y \int_{0}^{+\infty} z^{k-i} f\left(z, t \mid 0, t_{0}\right) d z,
\end{aligned}
$$

from which Equation (41) follows, recalling (20) and (38).

\section{References}

1. Bharucha-Reid, A.T. Elements of the Theory of Markov Processes and Their Applications; McGraw-Hill: New York, NY, USA, 1960.

2. Cox, D.R.; Miller, H.D. The Theory of Stochastic Processes; Chapman \& Hall/CRC: Boca Raton, FL, USA, 1996.

3. Ricciardi, L.M. Diffusion Processes and Related Topics in Biology; Springer: Berlin, Germany, 1977.

4. Ricciardi, L.M. Stochastic Population Theory: Diffusion Processes. In Mathematical Ecology; Hallam, T.G., Levin, S.A., Eds.; Springer: Berlin, Germany, 1986; Volume 17, pp. 191-238.

5. Tuckwell, H.C. Introduction to Theoretical Neurobiology: Volume 2, Nonlinear and Stochastic Theories; Cambridge University Press: Cambridge, MA, USA, 1988.

6. Gardiner, C.W. Handbook of Stochastic Methods: For Physics, Chemistry and the Natural Sciences; Springer: Berlin, Germany, 1985.

7. Feller, W. Diffusion processes in genetics. In Proceedings of the Second Berkeley Symposium on Mathematical Statistics and Probability; Statistical Laboratory of the University of California: Berkeley, CA, USA, 1951; pp. 227-246.

8. Ricciardi, L.M.; Di Crescenzo, A.; Giorno, V.; Nobile, A.G. An outline of theoretical and algorithmic approaches to first passage time problems with applications to biological modeling. Math. Jpn. 1999, 50, 247-322. 
9. Pugliese, A.; Milner, F. A structured population model with diffusion in structure space. J. Math. Biol. 2018, 77, $2079-2102$. [CrossRef]

10. Masoliver, J.; Perelló J. First-passage and escape problems in the Feller process. Phys. Rev. E 2012, 86, 041116. [CrossRef]

11. Masoliver, J. Nonstationary Feller process with time-varying coefficients. Phys. Rev. E 2016, 93, 012122. [CrossRef] [PubMed]

12. Di Crescenzo, A.; Nobile, A.G. Diffusion approximation to a queueing system with time-dependent arrival and service rates. Queueing Syst. 1995, 19, 41-62. [CrossRef]

13. Sacerdote, L. On the solution of the Fokker-Planck equation for a Feller process. Adv. Appl. Prob. 1990, 22, 101-110. [CrossRef]

14. Ditlevsen, S.; Lánský, P. Estimation of the input parameters in the Feller neuronal model. Phys. Rev. E 2006, 73, 061910. [CrossRef] [PubMed]

15. Buonocore, A.; Giorno, V.; Nobile, A.G.; Ricciardi, L.M. A neuronal modeling paradigm in the presence of refractoriness. BioSystems 2002, 67, 35-43. [CrossRef]

16. Giorno, V.; Lánský, P.; Nobile, A.G.; Ricciardi, L.M. Diffusion approximation and first-passage-time problem for a model neuron. III. A birth-and-death process approach. Biol. Cyber. 1988, 58, 387-404. [CrossRef]

17. Nobile, A.G.; Pirozzi, E. On time non-homogeneous Feller-type diffusion process in neuronal modeling. In Computer Aided Systems Theory_Eurocast 2015; Moreno-Díaz, R., Pichler, F., Eds.; Lecture Notes in Computer Science; Springer International Publishing: Cham, Switzerland, 2015; Volume 9520, pp. 183-191.

18. D’Onofrio, G.; Lánský, P.; Pirozzi, E. On two diffusion neuronal models with multiplicative noise: The mean first-passage time properties. Chaos 2018, 28, 043103. [CrossRef]

19. Tian, Y.; Zhang, H. Skew CIR process, conditional characteristic function, moments and bond pricing. Appl. Math. Comput. 2018, 329, 230-238. [CrossRef]

20. Cox, J.C.; Ingersoll, J.E., Jr.; Ross, S.A. A theory of the term structure of interest rates. Econometrica 1985, 53, 385-407. [CrossRef]

21. Maghsoodi, Y. Solution of the extended CIR term structure and bond option valuation. Math. Financ. 1996, 6, 89-109. [CrossRef]

22. Peng, Q.; Schellhorn, H. On the distribution of extended CIR model. Stat. Probab. Lett. 2018, 142, 23-29. [CrossRef]

23. Linetsky, V. Computing hitting time densities for CIR and OU diffusions. Applications to mean-reverting models. J. Comput. Financ. 2004, 7, 1-22. [CrossRef]

24. Di Nardo, E.; D'Onofrio, G. A cumulant approach for the first-passage-time problem of the Feller square-root process. Appl. Math. Comput. 2021, 391, 125707.

25. Lavigne, F.; Roques, L. Extinction times of an inhomogeneous Feller diffusion process: A PDF approach. Expo. Math. 2021, 39, 137-142. [CrossRef]

26. Giorno, V.; Nobile, A.G. Time-inhomogeneous Feller-type diffusion process with absorbing boundary condition. J. Stat. Phys. 2021 183, 1-27.

27. Bhattacharya, R.N.; Waymire, E.C. Stochastic Processes with Applications; Classics in Applied Mathematics; SIAM: Philadelphia, PA, USA, 2009.

28. Giorno, V.; Nobile, A.G. Bell polynomial approach for time-inhomogeneous linear birth-death process with immigration. Mathematics 2020, 8, 1123. [CrossRef]

29. Di Crescenzo, A.; Giorno, V.; Krishna Kumar, B.; Nobile, A.G. A double-ended queue with catastrophes and repairs, and a jump-diffusion approximation. Methodol. Comput. Appl. Probab. 2012, 14, 937-954. [CrossRef]

30. Di Crescenzo, A.; Giorno, V.; Krishna Kumar, B.; Nobile, A.G. A time-non-homogeneous double-ended queue with failures and repairs and its continuous approximation. Mathematics 2018, 6, 81. [CrossRef]

31. Dharmaraja, S.; Di Crescenzo, A.; Giorno, V.; Nobile, A.G. A continuous-time Ehrenfest model with catastrophes and its jump-diffusion approximation. J. Stat. Phys. 2015, 161, 326-345. [CrossRef]

32. Gan, X.; Waxman, D. Singular solution of the Feller diffusion equation via a spectral decomposition. Phys. Rev. E Stat. Nonlin. Soft. Matter Phys. 2015, 91, 012123. [CrossRef]

33. Abramowitz, I.A.; Stegun, M. Handbook of Mathematical Functions; Dover Publications, Inc.: New York, NY, USA, 1972.

34. Feller, W. Two singular diffusion problems. Ann. Math. 1951, 54, 173-182. [CrossRef]

35. Feller, W. The parabolic differential equations and the associated semi-groups of transformations. Ann. Math. 1952, 55, 468-518. [CrossRef]

36. Karlin, S.; Taylor, H.W. A Second Course in Stochastic Processes; Academic Press: New York, NY, USA, 1981.

37. Giorno, V.; Nobile, A.G.; Ricciardi, L.M.; Sacerdote, L. Some remarks on the Rayleigh process. J. Appl. Prob. 1986, 23, 398-408. [CrossRef]

38. Gradshteyn, I.S.; Ryzhik, I.M. Table of Integrals, Series and Products; Academic Press Inc.: Cambridge, MA, USA, 2014.

39. Erdèlyi, A.; Magnus, W.; Oberthettinger, F.; Tricomi, F.G. Higher Trascendental Functions; Mc Graw-Hill: New York, NY, USA, 1953; Volume II.

40. Coleman, B.D.; Hsieh Y.-H.; Knowles, G.P. On the optimal choice of $r$ for a population in a periodic environment. Math. Biosci. 1979, 46, 71-85. [CrossRef]

41. Keeling, M.J.; Rohani, P. Modeling Infectious Diseases in Humans and Animals;Princeton University Press: Princeton, NJ, USA, 2008.

42. Williams, W.E. Partial Differential Equations; Clarendon Press: Oxford, UK, 1980. 
43. Erdèlyi, A.; Magnus, W.; Oberthettinger, F.; Tricomi, F.G. Tables of Integral Transforms; Mc Graw-Hill: New York, NY, USA, 1954; Volume 1.

44. Comtet, L. Advanced Combinatorics: The Art of Finite and Infinite Expansions; D. Reidel Publishing Company: Dordrecht, The Netherlands; Boston, MA, USA, 1974. 